\title{
Stability of multidimensional undercompressive shock waves
}

\author{
JEAN-François COULOMBeL ${ }^{\dagger}$ \\ UMPA, CNRS - UMR 5669, École Normale Supérieure de Lyon, \\ 46 allée d'Italie, 69364 Lyon Cedex 07, France
}

[Received 13 June 2002 and in revised form 27 March 2003]

\begin{abstract}
This paper is devoted to the study of linear and nonlinear stability of undercompressive shock waves for first order systems of hyperbolic conservation laws in several space dimensions. We first recall the framework proposed by Freistühler to extend Majda's work on classical shock waves to undercompressive shock waves. Then we show how the so-called uniform stability condition yields a linear stability result in terms of a maximal $L^{2}$ estimate. We follow Majda's strategy on shock waves with several improvements and modifications inspired from Métivier's work. The linearized problems are solved by duality and the nonlinear equations by mean of a Newton type iteration scheme. Finally, we show how this work applies to phase transitions in an isothermal van der Waals fluid.
\end{abstract}

\section{Introduction}

In [14,13], Majda proved the (local in time) existence of multidimensional shock waves for systems of conservation laws. We refer to [15,21] for an overview of the method and its applications to gas dynamics. Majda's analysis dealt with shock waves satisfying Lax's entropy inequalities, and it relied on a uniform stability condition. His work was later refined by Métivier and Mokrane [16, 17], using new ideas of paradifferential calculus.

In several applications such as magnetohydrodynamics or the modeling of phase transitions, it happens that some shock waves violate Lax's entropy inequalities. Depending on their nature, these discontinuous solutions bear the name of undercompressive or overcompressive shock waves (we shall recall these notions later on). To select the physically relevant discontinuities, an additional admissibility criterion may be required (this is the case for undercompressive shock waves). For isothermal subsonic phase transitions, Slemrod and Truskinovsky independently derived an admissibility criterion based on the theory of capillarity [24, 25] (see also [22]). In this paper, we focus on undercompressive shocks and we shall follow a general approach, using an abstract admissibility criterion, as suggested by Freistühler. The analysis applies to some concrete problems (this approach was also used by Colombo and Corli in [6] for one-dimensional problems).

Our purpose is to establish the (local in time) existence of multidimensional undercompressive shock waves under a uniform stability assumption. This is the extension of Majda's work proposed by Freistühler in [10] (see also [9]). In particular, the uniform stability assumption stated below was already formulated in [10]. For technical reasons, we have adopted Métivier's approach and we shall often refer to [16] for detailed proofs. However, we emphasize that the analysis of [16] does not apply when solving the linearized equations (see Section 5 below), and we need here to

\footnotetext{
${ }^{\dagger}$ Email: jfcoulom@umpa.ens-lyon.fr
} 
make a specific assumption that is due to the undercompressive nature of the shocks we consider. This assumption is highly reasonable though and is met in our main example. This example deals with subsonic phase transitions in a van der Waals fluid. We are interested there in the existence of propagating interfaces separating liquid and vapor phases. Such an interface is not known in advance, and we thus face a free boundary problem. The evolution of this free boundary is governed by the Rankine-Hugoniot conditions and the visco-capillary admissibility criterion described below. In Section 6, we shall show how Freistühler's general framework, which we develop here, can be used to solve this specific problem of fluid dynamics. The stability of multidimensional dynamic phase transitions was first adressed by Benzoni-Gavage in [1, 2].

The remainder of this paper is organized as follows: in Section 2, we introduce the framework of our study and state our main assumptions. We recall the definition of undercompressive shock waves. In Section 3, we study the linear stability of a fixed planar undercompressive shock and show how the so-called uniform stability condition yields a maximal $L^{2}$ estimate. The study of planar shock waves is extended to the variable coefficients case in Section 4 In Section 5 , we shall detail how to solve the linearized equations as well as the nonlinear problem. In particular, we shall explain why Métivier's approach cannot be used. Finally, Section 6 is devoted to the application of our work to isothermal phase transitions in a van der Waals fluid.

\section{Description of the problem}

We consider a system of $n$ conservation laws in $\mathbb{R} \times \mathbb{R}^{d}$ :

$$
\sum_{j=0}^{d} \partial_{j} f_{j}(u)=0
$$

where $x_{0}$ is the time variable, also denoted by $t$ in what follows, $\left(x_{1}, \ldots, x_{d}\right)$ is the space variable and $\partial_{j}$ stands for the partial derivative with respect to $x_{j}$. The fluxes $f_{0}, \ldots, f_{d}$ are $\mathcal{C}^{\infty}$ functions defined on an open subset $U$ of $\mathbb{R}^{n}$ with values in $\mathbb{R}^{n}$. The jacobian matrix of $f_{j}$ at a point $u$ will be denoted by $A_{j}(u)$. For the sake of completeness, we assume that $f_{0}$ is a $\mathcal{C}^{\infty}$ diffeomorphism on its range. One can simply think of $f_{0}$ as the identity. We assume that the space dimension $d$ is strictly greater than 1 (that is, $d \geqslant 2$ ) and make the following hyperbolicity assumption:

Assumption 1 There exists a $\mathcal{C}^{\infty}$ mapping $S: U \rightarrow \mathcal{M}_{n}(\mathbb{R})$ such that

- $\forall j=0, \ldots, d, \forall u \in U, S(u) A_{j}(u)$ is symmetric,

- $\forall K$ compact $\subset U, \exists c_{K}>0$ such that $S(u) A_{0}(u) \geqslant c_{K} I$ for all $u \in K$.

Moreover, if we define the matrix $A(u, \xi)$ by

$$
\forall u \in U, \forall \xi \in \mathbb{R}^{d}, \quad A(u, \xi):=\sum_{j=1}^{d} \xi_{j} A_{0}(u)^{-1} A_{j}(u),
$$

then $A(u, \xi)$ is diagonalizable over $\mathbb{R}$ with $\mathcal{C}^{\infty}$ eigenvalues $\lambda_{1}, \ldots, \lambda_{m}$ (defined on $U \times \mathbb{R}^{d} \backslash\{0\}$ ) of fixed multiplicities $\alpha_{1}, \ldots, \alpha_{m}$. Without loss of generality, we may assume that

$$
\forall u \in U, \forall \xi \in \mathbb{R}^{d} \backslash\{0\}, \quad \lambda_{1}(u, \xi)<\cdots<\lambda_{m}(u, \xi) .
$$


We point out that Assumption 11 is easy to check on the system, unlike the block structure condition defined by Majda [14]. However, one could replace the last part of Assumption 1 (that is, the constant multiplicity assumption) by the more abstract block structure condition, as in [14, [16]. We also note that the equations of ideal magnetohydrodynamics are symmetrizable but violate Assumption 1 An open and interesting question is whether the block structure condition is satisfied by the MHD system. We refer to [4] for some results on shock waves in MHD.

We first recall a basic fact on piecewise smooth solutions to (1) (we refer to [20] for the proof). Let $\Gamma=\left\{x_{d}-\varphi\left(x_{0}, \ldots, x_{d-1}\right)=0\right\}$ be a smooth hypersurface in $\mathbb{R}^{d+1}$ and $u$ be a function smooth on either side of $\Gamma$. Then $u$ is a weak solution of (1) if and only if $u$ satisfies (1) (in the classical sense) on either side of $\Gamma$ and the Rankine-Hugoniot conditions hold at each point of $\Gamma$ :

$$
\forall \mathbf{x}=\left(x_{0}, \ldots, x_{d}\right) \in \Gamma, \quad \sum_{j=0}^{d-1} \partial_{j} \varphi\left[f_{j}(u)\right](\mathbf{x})-\left[f_{d}(u)\right](\mathbf{x})=0,
$$

the partial derivatives of $\varphi$ in the above formula being evaluated at $\left(x_{0}, \ldots, x_{d-1}\right)$. As usual we have let

$$
\left[f_{j}(u)\right](\mathbf{x}):=\lim _{s \rightarrow 0^{+}}\left(f_{j}(\mathbf{x}+s \mathbf{n})-f_{j}(\mathbf{x}-s \mathbf{n})\right) \quad \text { with } \quad \mathbf{n}:=\left(-\partial_{0} \varphi, \ldots,-\partial_{d-1} \varphi, 1\right)
$$

a normal vector to $\Gamma$ at point $\mathbf{x}$. In what follows, we omit the dependence on $\mathbf{x}$ in the expressions $\left[f_{j}(u)\right]$.

In this paper, we are interested in the stability of undercompressive shock waves. To fix ideas and notations, we consider a planar shock

$$
\mathbf{u}= \begin{cases}u_{r} & \text { if } x_{d}>\sigma t+v \cdot y \\ u_{l} & \text { if } x_{d}<\sigma t+v \cdot y\end{cases}
$$

which is a solution to (1). In (4), $u_{r}$ and $u_{l}$ are fixed vectors belonging to the open set $U, y=$ $\left(x_{1}, \ldots, x_{d-1}\right)$ is the vector formed by the tangential space coordinates, $v$ is a vector in $\mathbb{R}^{d-1}$ and $\sigma$ is the speed of propagation of the front. This corresponds to the equation

$$
\varphi\left(x_{0}, \ldots, x_{d-1}\right)=\sigma x_{0}+\sum_{j=1}^{d-1} v_{j} x_{j}
$$

for the shock front curve. Clearly $\mathbf{u}$ is a solution of (1) if and only if

$$
\sigma\left[f_{0}(\mathbf{u})\right]+\sum_{j=1}^{d-1} v_{j}\left[f_{j}(\mathbf{u})\right]=\left[f_{d}(\mathbf{u})\right] .
$$

Performing a rotation of the axes, we may assume $v=0$. Changing the last space variable $x_{d}$ to $x_{d}-\sigma t$, and correspondingly the flux $f_{d}$ to $f_{d}-\sigma f_{0}$, we may also assume that the shock is stationary, that is, $\sigma=0$ (this will simplify the statement of Assumption 2). Note that Assumption 1 is still satisfied after this change of observer. We assume that the shock $\mathbf{u}$ is noncharacteristic. More precisely, we make the following assumption: 
AsSUMPTION 2 There exist two integers $p, q \in\{1, \ldots, m\}$ such that

$$
\lambda_{q-1}\left(u_{l}, e_{d}\right)<0<\lambda_{q}\left(u_{l}, e_{d}\right) \text { and } \lambda_{p}\left(u_{r}, e_{d}\right)<0<\lambda_{p+1}\left(u_{r}, e_{d}\right) \text {, }
$$

where $e_{d}:=(0, \ldots, 0,1) \in \mathbb{R}^{d}$ is the vector of propagation of the shock wave $\mathbf{u}$.

If $q=1$ (resp. $p=m$ ) the first inequality on the left (resp. the last inequality on the right) is ignored. Assumption 2 is illustrated by Figure 1 where the characteristics entering the shock front curve $\left\{x_{d}=\sigma t\right\}$ are depicted (here we have not assumed $\sigma=0$ ).

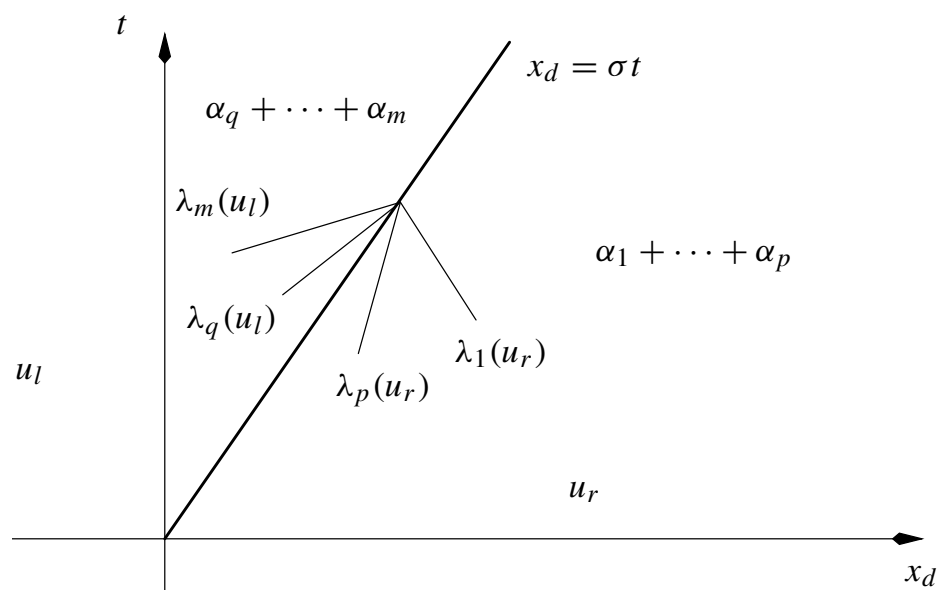

FIG. 1. Characteristics entering the shock front.

Following Freistühler [8], we define the number

$$
\kappa:=\left(\alpha_{q}+\cdots+\alpha_{m}\right)+\left(\alpha_{1}+\cdots+\alpha_{p}\right)-n,
$$

representing the amount by which the number of incoming characteristics (counted with multiplicity) exceeds $n$. We introduce the following terminology:

Definition 1 If $\kappa=1$ (that is, $p=q$ and $\lambda_{p}$ is a simple eigenvalue), then $\mathbf{u}$ is called a $p$-Lax shock wave.

If $\kappa<1$ (that is, $p<q$ ), then $\mathbf{u}$ is called an undercompressive shock wave.

If $\kappa>1$ (that is, $p>q$ ), then $\mathbf{u}$ is called an overcompressive shock wave.

From now on, we only consider the case of undercompressive shocks, that is, $\kappa \leqslant 0$. As already mentioned in some previous works (see e.g. [23] and references therein), in the case of an undercompressive shock as initial data, the associated Riemann problem has several solutions. In other words, the Rankine-Hugoniot conditions (3) are not sufficient to single out a unique weak piecewise smooth solution. Additional constraints on the shock $\mathbf{u}$ are needed. As explained in the introduction, we shall follow a general approach and will not discuss the way how the additional "admissibility criterion" is derived (except in Section 6 where we shall apply our results to phase transitions in van der Waals fluids). We thus fix, once for all, a $\mathcal{C}^{\infty}$ function $h: U \times U \times \mathbb{R} \times \mathbb{R}^{d-1} \rightarrow$ $\mathbb{R}^{N-n}$, where $N$ is an integer such that

$$
N-n=1-\kappa \geqslant 1 .
$$


We focus on the case where the additional constraints take the form

$$
h\left(u_{l}, u_{r}, \sigma, v\right)=0 .
$$

The integer $N-n$ counts the number of additional jump conditions that need to be prescribed and $N$ is the total amount of prescribed jump conditions including the Rankine-Hugoniot conditions. We now introduce the following definition:

DEFINITION 2 Let $\mathbf{u}$, defined by (4), be a planar undercompressive shock wave. We shall say that $\mathbf{u}$ is an admissible discontinuous solution if

$$
\sigma\left[f_{0}(\mathbf{u})\right]+\sum_{j=1}^{d-1} v_{j}\left[f_{j}(\mathbf{u})\right]-\left[f_{d}(\mathbf{u})\right]=0, \quad h\left(u_{l}, u_{r}, \sigma, v\right)=0 .
$$

Of course, there are many possible choices for $h$ (see e.g. Section 6), and our definition of admissible solutions heavily depends on the choice of $h$. The uniform stability assumption stated below also heavily depends on the choice of $h$. The reader is refered to [1,2] for a comparison of stability results according to the adopted criterion.

The (local in time) existence of admissible undercompressive shock waves is a free boundary problem. The function $\varphi$ defining the shock front is part of the unknown of the problem. To circumvent this difficulty, we introduce a change of variables in $\mathbb{R}^{d+1}$ with the formula

$$
\Phi\left(x_{0}, \ldots, x_{d}\right):=\left(x_{0}, \ldots, x_{d-1}, x_{d}+\varphi\left(x_{0}, \ldots, x_{d-1}\right)\right) .
$$

We have chosen here the standard change of variables, as in [14, 16, 17]. It maps the hyperplane $\left\{x_{d}=0\right\}$ onto the hypersurface $\Gamma$ and the two half-spaces $\left\{ \pm x_{d}>0\right\}$ on the two sides of $\Gamma$. We now perform a change of unknown functions. If $u$ is a smooth function on either side of $\Gamma$, then the function $u_{\sharp}$ defined by

$$
\forall\left(x_{0}, \ldots, x_{d}\right) \in \mathbb{R}^{d+1}, \quad u_{\sharp}\left(x_{0}, \ldots, x_{d}\right):=u\left(\Phi\left(x_{0}, \ldots, x_{d}\right)\right)
$$

is smooth on either side of the hyperplane $\left\{x_{d}=0\right\}$. It is now a straightforward application of the chain rule to verify that $u$ defines an admissible undercompressive shock if and only if

$$
\left\{\begin{array}{l}
L\left(u_{\sharp}^{ \pm}, \varphi\right) u_{\sharp}^{ \pm}=0, \quad \pm x_{d}>0, \\
B\left(u_{\sharp}^{+}, u_{\sharp}^{-}, \varphi\right)=0, \quad x_{d}=0,
\end{array}\right.
$$

where $u_{\sharp}^{+}$(resp. $u_{\sharp}^{-}$) denotes the restriction of $u_{\sharp}$ to the half-space $\left\{x_{d}>0\right\}$ (resp. $\left\{x_{d}<0\right\}$ ), and the operators $L$ and $B$ are defined by the following formulas:

$$
L(v, \psi) w:=\sum_{j=0}^{d-1} A_{j}(v) \partial_{j} w+\widetilde{A}_{d}(v, \nabla \psi) \partial_{d} w
$$

with

$$
\begin{gathered}
\widetilde{A}_{d}(v, \nabla \psi):=A_{d}(v)-\sum_{j=0}^{d-1} \partial_{j} \psi A_{j}(v), \\
B\left(w^{+}, w^{-}, \psi\right):=\left(\begin{array}{c}
\sum_{j=0}^{d-1} \partial_{j} \psi\left[f_{j}(w)\right]-\left[f_{d}(w)\right] \\
h\left(w^{-}, w^{+}, \partial_{0} \psi, \nabla_{y} \psi\right)
\end{array}\right) .
\end{gathered}
$$


Now that we have fixed the domain, the problem reduces to the following question: given an initial datum $u^{0}$ that is smooth on either side of a hypersurface $\left\{x_{d}=\varphi^{0}\left(x_{1}, \ldots, x_{d-1}\right)\right\}$, does there exist a solution $\left(u_{\sharp}, \varphi\right)$ of $(77)$ with initial value $\left(u_{\sharp}^{0}, \varphi^{0}\right)$, at least locally in time? Following Freistühler [10], we shall answer this question in the affirmative under a uniform stability assumption. This extends Majda's work to the framework set by Freistühler for undercompressive shocks. As in Majda's analysis, special attention will be paid to the linear stability of the reference undercompressive shock $\mathbf{u}$ with respect to small perturbations.

\section{The constant coefficient linearized system}

\subsection{The linearized equations}

We first examine the linear stability of the reference planar shock $\mathbf{u}(v=0$ and $\sigma=0)$. We introduce the linearized operators about the shock $\mathbf{u}$. Considering a family $u_{s}=\mathbf{u}+s v$ and $\varphi_{s}=s \psi$, we define the linear operators

$$
\mathcal{L}_{\mathbf{u}}\left(v^{ \pm}, \psi\right):=\left.\frac{\mathrm{d}}{\mathrm{d} s} L\left(u_{s}^{ \pm}, \varphi_{s}\right) u_{s}^{ \pm}\right|_{s=0}, \quad \mathcal{B}_{\mathbf{u}}(v, \psi):=\left.\frac{\mathrm{d}}{\mathrm{d} s} B\left(u_{s}^{+}, u_{s}^{-}, \varphi_{s}\right)\right|_{s=0} .
$$

Since $\mathbf{u}$ is constant on either side of $\left\{x_{d}=0\right\}$, the linearized operators read

$$
\begin{array}{r}
\mathcal{L}_{\mathbf{u}}\left(v^{ \pm}, \psi\right)=\mathcal{L}_{\mathbf{u}} v^{ \pm}=\sum_{j=0}^{d} A_{j}\left(u_{r, l}\right) \partial_{j} v^{ \pm}, \quad \pm x_{d}>0, \\
\mathcal{B}_{\mathbf{u}}(v, \psi)=\left(\begin{array}{c}
\sum_{j=0}^{d-1} \partial_{j} \psi\left[f_{j}(\mathbf{u})\right]-A_{d}\left(u_{r}\right) v^{+}+A_{d}\left(u_{l}\right) v^{-} \\
\partial_{\sigma} h \partial_{0} \psi+d_{v} h \cdot \nabla_{y} \psi+d_{u_{r}} h \cdot v^{+}+d_{u_{l}} h \cdot v^{-}
\end{array}\right), \quad x_{d}=0 .
\end{array}
$$

We let

$$
b_{0}(\mathbf{u}):=\left(\begin{array}{c}
{\left[f_{0}(\mathbf{u})\right]} \\
\partial_{\sigma} h
\end{array}\right), \quad b_{j}(\mathbf{u}):=\left(\begin{array}{c}
{\left[f_{j}(\mathbf{u})\right]} \\
\partial_{\nu_{j}} h
\end{array}\right), \quad 1 \leqslant j \leqslant d-1,
$$

and

$$
M(\mathbf{u})\left(\begin{array}{c}
v^{+} \\
v^{-}
\end{array}\right):=\left(\begin{array}{c}
-A_{d}\left(u_{r}\right) \\
d_{u_{r}} h
\end{array}\right) v^{+}+\left(\begin{array}{c}
A_{d}\left(u_{l}\right) \\
d_{u_{l}} h
\end{array}\right) v^{-} .
$$

Then the linearized boundary value operator $\mathcal{B}_{\mathbf{u}}$ reads

$$
\mathcal{B}_{\mathbf{u}}(v, \psi)=\sum_{j=0}^{d-1} \partial_{j} \psi b_{j}(\mathbf{u})+M(\mathbf{u})\left(\begin{array}{l}
v^{+} \\
v^{-}
\end{array}\right)
$$

We are now led to considering the following boundary value problem for the unknown functions $\left(v^{ \pm}, \psi\right)$ :

$$
\left\{\begin{array}{l}
\mathcal{L}_{\mathbf{u}} v^{ \pm}=f_{ \pm}, \quad \pm x_{d}>0 \\
\mathcal{B}_{\mathbf{u}}(v, \psi)=g, \quad x_{d}=0
\end{array}\right.
$$

where the source terms $f_{ \pm}$and $g$ belong to appropriate function spaces. 
Note that $(9]$ is a constant coefficient hyperbolic boundary value problem; moreover, the boundary $\left\{x_{d}=0\right\}$ is noncharacteristic because of Assumption 2 both matrices $A_{d}\left(u_{r}\right)$ and $A_{d}\left(u_{l}\right)$ are nonsingular. Kreiss's theory [12] does not apply directly because the boundary conditions involve partial derivatives of $\psi$. Nonetheless, we attack $(9)$ by the same kind of arguments. Formally, we perform a Laplace transform in $x_{0}$ and a Fourier transform in the tangential space variables $\left(x_{1}, \ldots, x_{d-1}\right)$. We also make the change of variables $\left(x_{d} \mapsto-x_{d}\right)$ in the evolution equation for $v^{-}$. This yields the following system of ODEs:

$$
\begin{aligned}
& \left(\tau A_{0}\left(u_{r}\right)+i \sum_{j=1}^{d-1} \eta_{j} A_{j}\left(u_{r}\right)\right) V^{+}+A_{d}\left(u_{r}\right) \frac{\mathrm{d} V^{+}}{\mathrm{d} x_{d}}=F_{+}, \\
& \left(\tau A_{0}\left(u_{l}\right)+i \sum_{j=1}^{d-1} \eta_{j} A_{j}\left(u_{l}\right)\right) V^{-}-A_{d}\left(u_{l}\right) \frac{\mathrm{d} V^{-}}{\mathrm{d} x_{d}}=F_{-},
\end{aligned}
$$

in the domain $\left\{x_{d}>0\right\}$, with the boundary conditions:

$$
\left(\tau b_{0}(\mathbf{u})+i \sum_{j=1}^{d-1} \eta_{j} b_{j}(\mathbf{u})\right) \Psi+M(\mathbf{u})\left(\begin{array}{l}
V^{+}(0) \\
V^{-}(0)
\end{array}\right)=G,
$$

on $\left\{x_{d}=0\right\}$. The complex number $\tau=\gamma+i \eta_{0}$ has nonnegative real part. In what follows, we shall denote by $\eta$ the vector $\left(\eta_{0}, \eta_{1}, \ldots, \eta_{d-1}\right) \in \mathbb{R}^{d}$.

Because the boundary is noncharacteristic, we may rewrite systems $10 \mathrm{a}-10 \mathrm{~b})$ as an ODE system of the form

$$
\begin{gathered}
\frac{\mathrm{d}}{\mathrm{d} x_{d}}\left(\begin{array}{l}
V^{+} \\
V^{-}
\end{array}\right)=\mathcal{A}(\mathbf{u}, \eta, \gamma)\left(\begin{array}{l}
V^{+} \\
V^{-}
\end{array}\right)+\mathcal{A}_{d}(\mathbf{u})^{-1}\left(\begin{array}{l}
F_{+} \\
F_{-}
\end{array}\right) \\
\text {with } \mathcal{A}(\mathbf{u}, \eta, \gamma):=\left(\begin{array}{cc}
\mathcal{A}_{r}(\mathbf{u}, \eta, \gamma) & \mathbf{0} \\
\mathbf{0} & \mathcal{A}_{l}(\mathbf{u}, \eta, \gamma)
\end{array}\right) .
\end{gathered}
$$

Matrices $\mathcal{A}_{r, l}$ and $\mathcal{A}_{d}$ in (11) are defined by

$$
\begin{aligned}
\mathcal{A}_{r, l}(\mathbf{u}, \eta, \gamma) & :=\mp A_{d}\left(u_{r, l}\right)^{-1}\left(\gamma A_{0}\left(u_{r, l}\right)+i \sum_{j=0}^{d-1} \eta_{j} A_{j}\left(u_{r, l}\right)\right), \\
\mathcal{A}_{d}(\mathbf{u}) & :=\left(\begin{array}{cc}
A_{d}\left(u_{r}\right) & \mathbf{0} \\
\mathbf{0} & -A_{d}\left(u_{l}\right)
\end{array}\right) .
\end{aligned}
$$

Defining

we also have

$$
\mathcal{A}_{j}(\mathbf{u}):=\left(\begin{array}{cc}
A_{j}\left(u_{r}\right) & \mathbf{0} \\
\mathbf{0} & A_{j}\left(u_{l}\right)
\end{array}\right),
$$

$$
\mathcal{A}(\mathbf{u}, \eta, \gamma):=-\mathcal{A}_{d}(\mathbf{u})^{-1}\left(\gamma \mathcal{A}_{0}(\mathbf{u})+i \sum_{j=0}^{d-1} \eta_{j} \mathcal{A}_{j}(\mathbf{u})\right) .
$$

Finally, we define the symbol associated with the shock front:

$$
b(\mathbf{u}, \eta, \gamma):=\gamma b_{0}(\mathbf{u})+i \sum_{j=0}^{d-1} \eta_{j} b_{j}(\mathbf{u}) .
$$


As noted by Hersh [11], the homogeneous part of the ODEs (11) is hyperbolic when $\gamma>0$, that is, the matrix $\mathcal{A}(\mathbf{u}, \eta, \gamma)$ does not admit purely imaginary eigenmodes when $\gamma>0$. For $\gamma>0$, we define $\mathcal{E}^{-}(\eta, \gamma)$ as the stable subspace of $\mathcal{A}$, that is, the set of boundary values at $x_{d}=0$ of solutions to (11) that are square integrable on $\left[0, \infty\left[\right.\right.$ when $F_{+}=F_{-}=0$. The dimension of $\mathcal{E}^{-}(\eta, \gamma)$ is equal to the dimension of $\mathcal{E}^{-}(0,1)$ for all $(\eta, \gamma), \gamma>0$ (this is just a continuity/connectedness argument). To compute the dimension of $\mathcal{E}^{-}(0,1)$, we seek the eigenmodes of $\mathcal{A}(\mathbf{u}, 0,1)$. These are the roots $\omega$ of the dispersion equation

$$
\operatorname{det}[\omega I-\mathcal{A}(\mathbf{u}, 0,1)]=0 .
$$

The definition of $\mathcal{A}$ shows that $\omega I-\mathcal{A}(\mathbf{u}, 0,1)$ is singular if and only if $A_{0}\left(u_{r}\right)+\omega A_{d}\left(u_{r}\right)$ or $A_{0}\left(u_{l}\right)-\omega A_{d}\left(u_{l}\right)$ is singular. As a consequence, $\omega$ satisfies the dispersion equation if and only if there exists an integer $k \in\{1, \ldots, m\}$ such that

$$
\lambda_{k}\left(u_{r}, e_{d}\right) \omega=-1 \quad \text { or } \quad \lambda_{k}\left(u_{l}, e_{d}\right) \omega=1,
$$

where $e_{d}=(0, \ldots, 0,1) \in \mathbb{R}^{d}$. Assumption 2 shows that such values of $\omega$ are negative for $k=$ $p+1, \ldots, m$ in the first case and $k=1, \ldots, q-1$ in the second case. Taking multiplicities into account, this shows that $\mathcal{E}^{-}(0,1)$ has dimension $n-\kappa$, with $\kappa$ defined by $(5)$.

For fixed $\eta \neq 0$, the stable subspace $\mathcal{E}^{-}(\eta, \gamma)$ admits a continuous extension to $(\eta, 0)$ (see [3]); we still denote this extension by $\mathcal{E}^{-}(\eta, 0)$. However, for $\gamma=0$, vectors in the extended stable subspace do not always correspond to boundary values of square integrable functions because of the possible occurrence of purely imaginary eigenmodes. This is widely detailed in [1,7, 14] for Euler's equations of isentropic gas dynamics.

We define the hemisphere of $\mathbb{R}^{d+1}$ as

$$
\Sigma:=\left\{(\eta, \gamma) \in \mathbb{R}^{d+1}: \gamma^{2}+|\eta|^{2}=1 \text { and } \gamma \geqslant 0\right\}
$$

Following Freistühler [10], we make the following uniform stability assumption:

ASSUMPTION 3 (Uniform stability) There exists a positive constant $c>0$ such that for all $(\eta, \gamma) \in \Sigma$,

$$
\forall(\chi, Z) \in \mathbb{C} \times \mathcal{E}^{-}(\eta, \gamma), \quad|\chi b(\mathbf{u}, \eta, \gamma)+M(\mathbf{u}) Z| \geqslant c(|\chi|+|Z|)
$$

By compactness of $\Sigma$, Assumption 3 is equivalent to the requirement that for all $(\eta, \gamma) \in \Sigma$, the subspace

$$
\left\{(\chi, Z) \in \mathbb{C} \times \mathcal{E}^{-}(\eta, \gamma): \chi b(\mathbf{u}, \eta, \gamma)+M(\mathbf{u}) Z=0\right\}
$$

is reduced to $\{0\}$. This is true because the mapping

$$
(\chi, Z) \in \mathbb{C} \times \mathbb{C}^{2 n} \mapsto \chi b(\mathbf{u}, \eta, \gamma)+M(\mathbf{u}) Z
$$

has values in $\mathbb{C}^{N}$, and $N=n-\kappa+1$ is the dimension of $\mathbb{C} \times \mathcal{E}^{-}$. Assumption 3 is therefore the direct extension of Majda's uniform stability condition to undercompressive shock waves, once additional jump conditions have been prescribed.

In the next subsection, we show that Assumption 3 yields a maximal $L^{2}$ energy estimate for the linearized problem (9). 


\subsection{Stability of a planar undercompressive shock}

In order to state our first result, we need to introduce a few notations. Denote by $\Omega$ and $\omega$ the domains

$$
\Omega:=\mathbb{R}_{+}^{d+1}=\left\{\left(x_{0}, \ldots, x_{d}\right) \in \mathbb{R}^{d+1}: x_{d}>0\right\} \quad \text { and } \quad \omega:=\mathbb{R}^{d}=\partial \Omega .
$$

For $\gamma>0$ and $s \in \mathbb{R}$ we define the following symbols:

$$
\forall \xi \in \mathbb{R}^{d}, \quad \lambda^{s, \gamma}(\xi):=\left(\gamma^{2}+|\xi|^{2}\right)^{s / 2} .
$$

The usual Sobolev spaces $H^{s}\left(\mathbb{R}^{d}\right)$ are equipped with the weighted norms (depending on the positive parameter $\gamma$ )

$$
\|v\|_{s, \gamma}^{2}:=\frac{1}{(2 \pi)^{d}} \int_{\mathbb{R}^{d}} \lambda^{2 s, \gamma}(\xi)|\widehat{v}(\xi)|^{2} \mathrm{~d} \xi .
$$

These weighted norms enable one to construct a parameter version of the classical pseudodifferential calculus that is of constant use in the study of initial boundary value problems for hyperbolic systems (see e.g. [5, 12, 14]).

The space $L^{2}(\Omega)$ is equipped with the usual norm

$$
\|v\|_{0}^{2}:=\int_{\Omega}|v(z)|^{2} \mathrm{~d} z
$$

The Laplace transform performed in the normal modes analysis amounts to working with the new functions $\tilde{v}:=\exp (-\gamma t) v$ and $\widetilde{\psi}:=\exp (-\gamma t) \psi, \gamma>0$. This leads to the introduction of the weighted operators:

$$
\mathcal{L}_{\mathbf{u}}^{\gamma} \widetilde{v}:=\mathcal{L}_{\mathbf{u}} \widetilde{v}+\gamma \mathcal{A}_{0}(\mathbf{u}) \widetilde{v}, \quad \mathcal{B}_{\mathbf{u}}^{\gamma}(\widetilde{v}, \widetilde{\psi}):=\mathcal{B}_{\mathbf{u}}(\widetilde{v}, \widetilde{\psi})+\gamma \widetilde{\psi} b_{0}(\mathbf{u})
$$

One easily checks that $(9)$ is equivalent to

$$
\begin{cases}\mathcal{L}_{\mathbf{u}}^{\gamma} \widetilde{v}=\exp (-\gamma t) f & \text { for } x_{d}>0 \\ \mathcal{B}_{\mathbf{u}}^{\gamma}(\widetilde{v}, \widetilde{\psi})=\exp (-\gamma t) g & \text { for } x_{d}=0\end{cases}
$$

For convenience, we drop the tilde from $\widetilde{v}$ and $\widetilde{\psi}$. The basic estimate is the same as for uniformly stable Lax shocks:

THEOREM 1 Let $\mathbf{u}$ be an admissible planar shock satisfying Assumption 3 . Then there exists a constant $C>0$ such that for all $v \in H^{1}(\Omega)$, for all $\psi \in H^{1}(\omega)$ and for all $\gamma \geqslant 1$, the following estimate holds:

$$
\gamma\|v\|_{0}^{2}+\left\|v_{\left.\right|_{x_{d}=0}}\right\|_{0}^{2}+\|\psi\|_{1, \gamma}^{2} \leqslant C\left(\frac{1}{\gamma}\left\|\mathcal{L}_{\mathbf{u}}^{\gamma} v\right\|_{0}^{2}+\left\|\mathcal{B}_{\mathbf{u}}^{\gamma}(v, \psi)\right\|_{0}^{2}\right) .
$$

Energy estimates in Sobolev spaces of higher order are also available, provided that $v$ and $\psi$ are sufficiently regular.

To prove Theorem 1, one uses a Kreiss symmetrizer. This is possible because the boundary conditions satisfy an analogue of the uniform Lopatinskiı̌ condition. We shall not detail the proof, and refer the interested reader to [5, 7, 12, 16]. 


\section{The variable coefficient linearized problem}

It is crucial in the proof of nonlinear stability of a planar shock to obtain an estimate of the same type as (13) for the linearized operators about interfaces that are close to but different from hyperplanes. The proof is entirely similar to Métivier's, so we omit it. We shall only give some indications and references.

We keep the same $\mathbf{u}$ as in the preceding section. There exists an open set $\mathcal{U}$ in $\mathbb{R}^{n} \times \mathbb{R}^{n} \times \mathbb{R} \times \mathbb{R}^{d-1}$ containing $(\mathbf{0}, \mathbf{0}, 0, \mathbf{0})$ such that for all $\left(w_{r}, w_{l}, \sigma, v\right) \in \mathcal{U}$, one has

$$
\begin{aligned}
u_{l}+w_{l} \in U, & \lambda_{q-1}\left(u_{l}+w_{l}, \xi\right)<\sigma<\lambda_{q}\left(u_{l}+w_{l}, \xi\right), \\
u_{r}+w_{r} \in U, & \lambda_{p}\left(u_{r}+w_{r}, \xi\right)<\sigma<\lambda_{p+1}\left(u_{r}+w_{r}, \xi\right),
\end{aligned}
$$

where $\xi:=(-v, 1) \in \mathbb{R}^{d}$. In other words, $\mathcal{U}$ is an open set such that all planar shocks associated with elements of $\mathcal{U}$ are noncharacteristic. Furthermore, one can choose $\mathcal{U}$ such that all planar shocks associated with elements of $\mathcal{U}$ are uniformly stable, that is, satisfy Assumption 3 We fix a compact subset $\mathcal{K} \subset \mathcal{U}$ and consider mappings $\dot{u}_{r}, \dot{u}_{l}, \varphi$ such that $\varphi$ is defined on $\mathbb{R}^{d}, \dot{u}_{r}$ (resp. $\dot{u}_{l}$ ) is defined on $\left\{x_{d} \geqslant \varphi\left(x_{0}, \ldots, x_{d-1}\right)\right\}$ (resp. $\left.\left\{x_{d} \leqslant \varphi\left(x_{0}, \ldots, x_{d-1}\right)\right\}\right)$ and $\left(\dot{u}_{r}, \dot{u}_{l}, \nabla \varphi\right)$ takes its values in $\mathcal{K}$. We define a function

$$
\mathbf{a}:= \begin{cases}u_{r}+\dot{u}_{r}(\mathbf{x}) & \text { if } x_{d}>\varphi\left(x_{0}, \ldots, x_{d-1}\right), \\ u_{l}+\dot{u}_{l}(\mathbf{x}) & \text { if } x_{d}<\varphi\left(x_{0}, \ldots, x_{d-1}\right) .\end{cases}
$$

The regularity of $\dot{u}_{r}, \dot{u}_{l}$ and $\varphi$ has not been specified yet. One can think of them as smooth perturbations of the stationary shock wave $\mathbf{u}$ (some kind of first order correction in an asymptotic expansion). We shall be more precise below.

With a slight abuse of notation, we often use the shorthand a to denote all functions $\left(\dot{u}_{r}, \dot{u}_{l}, \varphi\right)$, but we point out that we shall linearize both operators $L$ and $B$ with respect to $u$ and $\varphi$. More precisely, we consider a family $u_{s}=\mathbf{a}_{\sharp}+s v$ and $\varphi_{s}=\varphi+s \psi$. Then we define

$$
\begin{aligned}
\mathcal{L}_{\mathbf{a}}\left(v^{ \pm}, \psi\right) & :=\left.\frac{\mathrm{d}}{\mathrm{d} s} L\left(u_{s}^{ \pm}, \varphi_{s}\right) u_{s}^{ \pm}\right|_{s=0}, \\
\mathcal{B}_{\mathbf{a}}(v, \psi) & :=\left.\frac{\mathrm{d}}{\mathrm{d} s} B\left(u_{s}^{+}, u_{s}^{-}, \varphi_{s}\right)\right|_{s=0} .
\end{aligned}
$$

Recall that $\mathbf{a}_{\sharp}$ is the function deduced from a after the change of variables $\Phi$. A direct computation shows that

$$
\begin{aligned}
& \mathcal{L}_{\mathbf{a}}\left(v^{ \pm}, \psi\right)=\sum_{j=0}^{d-1} A_{j}\left(\mathbf{a}_{\sharp}^{ \pm}\right) \partial_{j} v^{ \pm}+\sum_{j=0}^{d-1}\left(d A_{j}\left(\mathbf{a}_{\sharp}^{ \pm}\right) \cdot v^{ \pm}\right) \partial_{j} \mathbf{a}_{\sharp}^{ \pm}+\widetilde{A}_{d}\left(\mathbf{a}_{\sharp}^{ \pm}, \nabla \varphi\right) \partial_{d} v^{ \pm} \\
& -\sum_{j=0}^{d-1} \partial_{j} \psi A_{j}\left(\mathbf{a}_{\sharp}^{ \pm}\right) \partial_{d} \mathbf{a}_{\sharp}^{ \pm}+\left(d_{u} \widetilde{A}_{d}\left(\mathbf{a}_{\sharp}^{ \pm}, \nabla \varphi\right) \cdot v^{ \pm}\right) \partial_{d} \mathbf{a}_{\sharp}^{ \pm}, \quad \quad \pm x_{d}>0,
\end{aligned}
$$

and

$$
\mathcal{B}_{\mathbf{a}}(v, \psi)=\left(\begin{array}{c}
\sum_{j=0}^{d-1} \partial_{j} \psi\left[f_{j}\left(\mathbf{a}_{\sharp}\right)\right]-\widetilde{A}_{d}\left(\mathbf{a}_{\sharp}^{+}, \nabla \varphi\right) v^{+}+\widetilde{A}_{d}\left(\mathbf{a}_{\sharp}^{-}, \nabla \varphi\right) v^{-} \\
\partial_{\sigma} h \partial_{0} \psi+d_{\nu} h \cdot \nabla_{y} \psi+d_{u_{r}} h \cdot v^{+}+d_{u_{l}} h \cdot v^{-}
\end{array}\right), \quad x_{d}=0 .
$$


Here, the partial derivatives of $h$ are evaluated at the point $\left(\mathbf{a}_{\sharp}^{-}, \mathbf{a}_{\sharp}^{+}, \nabla \varphi\right)$. We decompose the linearized operator $\mathcal{L}_{\mathrm{a}}$ as

$$
\mathcal{L}_{\mathbf{a}}\left(v^{ \pm}, \psi\right)=L\left(\mathbf{a}_{\sharp}^{ \pm}, \varphi\right) v^{ \pm}-\sum_{j=0}^{d-1} \partial_{j} \psi A_{j}\left(\mathbf{a}_{\sharp}^{ \pm}\right) \partial_{d} \mathbf{a}_{\sharp}^{ \pm}+E\left(\mathbf{a}_{\sharp}^{ \pm}, \varphi\right) v^{ \pm}
$$

where $L$ is defined by $(8 \mathrm{a})-(8 \mathrm{~b})$ and

$$
E\left(\mathbf{a}_{\sharp}^{ \pm}, \varphi\right) v^{ \pm}:=\sum_{j=0}^{d-1}\left(d A_{j}\left(\mathbf{a}_{\sharp}^{ \pm}\right) \cdot v^{ \pm}\right) \partial_{j} \mathbf{a}_{\sharp}^{ \pm}+\left(d_{u} \widetilde{A}_{d}\left(\mathbf{a}_{\sharp}^{ \pm}, \nabla \varphi\right) \cdot v^{ \pm}\right) \partial_{d} \mathbf{a}_{\sharp}^{ \pm}
$$

is the zero order part (in $v^{ \pm}$) of $\mathcal{L}_{\mathbf{a}}$. The principal part of $\mathcal{L}_{\mathbf{a}}$ is obtained by dropping the terms in $\psi$ and the zero order terms in $v^{ \pm}$in the expression of $\mathcal{L}_{\mathbf{a}}$, and thus coincides with the operator $L\left(\mathbf{a}_{ \pm}^{ \pm}, \varphi\right)$. With little risk of confusion, we shall still denote by $\mathcal{L}_{\mathbf{a}}$ the principal part of this operator. From now on, we only consider the principal part of the linearized operators. With the same notations as in the preceeding section, the (principal part of the) linearized equations read

$$
\begin{cases}\mathcal{L}_{\mathbf{a}} v=\sum_{j=0}^{d} \mathcal{A}_{j}(\mathbf{a}) \partial_{j} v=f, & x_{d}>0, \\ \mathcal{B}_{\mathbf{a}}(v, \psi)=\sum_{j=0}^{d-1} \partial_{j} \psi b_{j}(\mathbf{a})+M(\mathbf{a}) v=g, & x_{d}=0,\end{cases}
$$

once we define $v:=\left(v^{+}, v^{-}\right)$and perform the change of variable $x_{d} \mapsto-x_{d}$ in the evolution equation for $v^{-}$. Our goal is to obtain an $L^{2}$ estimate for the weighted system:

$$
\begin{cases}\mathcal{L}_{\mathbf{a}}^{\gamma} v:=\mathcal{L}_{\mathbf{a}} v+\gamma \mathcal{A}_{0}(\mathbf{a}) v=\exp (-\gamma t) f, & x_{d}>0 \\ \mathcal{B}_{\mathbf{a}}^{\gamma}(v, \psi):=\mathcal{B}_{\mathbf{a}}(v, \psi)+\gamma \psi b_{0}(\mathbf{a})=\exp (-\gamma t) g, & x_{d}=0 .\end{cases}
$$

To quantify our results, we fix a constant $K>0$ and assume that

$$
\|\nabla \varphi\|_{W^{1, \infty}\left(\mathbb{R}^{d}\right)} \leqslant K, \quad\left\|\left(\dot{u}_{r}, \dot{u}_{l}\right)\right\|_{W^{1, \infty}(\Omega)} \leqslant K .
$$

Then we have the following result:

THEOREM 2 Assume that $\left(\dot{u}_{r, l}, \nabla \varphi\right)$ takes its values in a compact subset $\mathcal{K} \subset \mathcal{U}$. Then there exist two constants $C(K)>0$ and $\gamma_{0} \geqslant 1$, depending only on $K$ and $\mathcal{K}$, such that for all $v \in H^{1}(\Omega)$ and all $\psi \in H^{1}(\omega)$, the following estimate holds:

$$
\forall \gamma \geqslant \gamma_{0}, \quad \gamma\|v\|_{0}^{2}+\left\|v_{\left.\right|_{x_{d}=0}}\right\|_{0}^{2}+\|\psi\|_{1, \gamma}^{2} \leqslant C(K)\left(\frac{1}{\gamma}\left\|\mathcal{L}_{\mathbf{a}}^{\gamma} v\right\|_{0}^{2}+\left\|\mathcal{B}_{\mathbf{a}}^{\gamma}(v, \psi)\right\|_{0}^{2}\right),
$$

where the operators $\mathcal{L}_{\mathbf{a}}^{\gamma}$ and $\mathcal{B}_{\mathbf{a}}^{\gamma}$ are the principal parts of the linearized operators, as defined in (16).

Theorem 2 is proved as in [16], using the Bony-Meyer paradifferential calculus. This technique enables us to circumvent the difficulty of Lipschitzean coefficients. It also enables us to control the constant $C(K)$, and to show that it only depends on $K$. We refer the reader to [16,7] for a complete proof which we omit here. 


\section{Well-posedness of the linearized problem}

In this section, we are going to solve the linearized equations (15) supplemented with an initial condition. Recall that the linearized operators have the following form:

$$
\begin{aligned}
\mathcal{L}_{\mathbf{a}} v=\sum_{j=0}^{d} \mathcal{A}_{j}(\mathbf{a}) \partial_{j} v, & x_{d}>0, \\
\mathcal{B}_{\mathbf{a}}(v, \psi)=\sum_{j=0}^{d-1} \partial_{j} \psi b_{j}(\mathbf{a})+M(\mathbf{a}) v, & x_{d}=0 .
\end{aligned}
$$

We first solve the boundary value problem

$$
\begin{cases}\mathcal{L}_{\mathbf{a}} v=f, & x_{d}>0, \\ \mathcal{B}_{\mathbf{a}}(v, \psi)=g, & x_{d}=0,\end{cases}
$$

with no initial condition and data $f$ and $g$ in weighted spaces. This is done with the help of a suitable dual problem that we introduce in the next subsection. In [16], Métivier uses a symmetric form of the equations. These arguments cannot be used here because of the undercompressive character of our shock waves (see especially the proof of Lemma 3.2.1 in [16]). Some modifications are required. They are detailed in the next subsection.

\subsection{The dual problem. Basic properties}

The symbol $b$ defined by (12) will not be used any more, so with little risk of confusion, we define the matrix

$$
b(\mathbf{a}):=\left(\begin{array}{lll}
b_{0}(\mathbf{a}) & \cdots & \left.b_{d-1}(\mathbf{a})\right) .
\end{array}\right.
$$

With this definition, we have

$$
\mathcal{B}_{\mathbf{a}}(v, \psi)=b(\mathbf{a}) \nabla \psi+M(\mathbf{a}) v .
$$

Moreover, the uniform stability condition implies that the matrix $b(\mathbf{a})$ is one-to-one for all $\mathbf{a}=$ $\left(\dot{u}_{r}, \dot{u}_{l}, \nabla \varphi\right) \in \mathcal{U}$. As a consequence, for $\mathcal{U}$ small enough, there exists a $\mathcal{C}^{\infty}$ mapping $P: \mathcal{U} \rightarrow$ $G l_{N}(\mathbb{R})$ such that

$$
\forall \mathbf{a} \in \mathcal{U}, \quad P(\mathbf{a}) b(\mathbf{a})=\left(\begin{array}{c}
I_{d} \\
\mathbf{0}
\end{array}\right) .
$$

The boundary conditions in (18) equivalently read

$$
\left(\begin{array}{c}
\nabla \psi \\
\mathbf{0}
\end{array}\right)+P(\mathbf{a}) M(\mathbf{a}) v=P(\mathbf{a}) g .
$$

We make the following (reasonable) assumption:

Assumption 4 The matrix $M(\mathbf{u}) \in \mathcal{M}_{N, 2 n}(\mathbb{R})$ has maximal rank.

When dealing with Lax shock waves, the analogue of Assumption 4 is automatically satisfied because the shock wave is noncharacteristic (see [16]).

Up to shrinking $\mathcal{U}$, the matrix $M(\mathbf{a})$ has still maximal rank for all $\mathbf{a}=\left(\dot{u}_{r}, \dot{u}_{l}, \nabla \varphi\right) \in \mathcal{U}$ and we have the following lemma: 
LEMma 1 There exists a $\mathcal{C}^{\infty}$ mapping $N: \mathcal{U} \rightarrow \mathcal{M}_{2 n-N, 2 n}(\mathbb{R})$ such that for all a $\in \mathcal{U}$,

$$
\mathbb{R}^{2 n}=\operatorname{Ker} P(\mathbf{a}) M(\mathbf{a}) \oplus \operatorname{Ker} N(\mathbf{a}) .
$$

Moreover, there exist two $\mathcal{C}^{\infty}$ mappings

$$
M^{\prime}: \mathcal{U} \rightarrow \mathcal{M}_{2 n, N}(\mathbb{R}) \quad \text { and } \quad N^{\prime}: \mathcal{U} \rightarrow \mathcal{M}_{2 n, 2 n-N}(\mathbb{R})
$$

such that for all $\mathbf{a} \in \mathcal{U}$,

$$
I_{2 n}=M^{\prime}(\mathbf{a}) P(\mathbf{a}) M(\mathbf{a})+N^{\prime}(\mathbf{a}) N(\mathbf{a}) .
$$

Proof. Because the matrix $P(\mathbf{a}) M(\mathbf{a})$ has maximal rank for all a $\in \mathcal{U}$, we can choose $N(\mathbf{a}) \in$ $\mathcal{M}_{2 n-N, 2 n}(\mathbb{R})$, depending smoothly on $\mathbf{a} \in \mathcal{U}$, such that the matrix

$$
\left(\begin{array}{c}
P(\mathbf{a}) M(\mathbf{a}) \\
N(\mathbf{a})
\end{array}\right)
$$

is invertible for all $\mathbf{a} \in \mathcal{U}$. Such a matrix $N(\mathbf{a})$ automatically satisfies

$$
\mathbb{R}^{2 n}=\operatorname{Ker} P(\mathbf{a}) M(\mathbf{a}) \oplus \operatorname{Ker} N(\mathbf{a}) .
$$

Moreover, $N(\mathbf{a})$ has maximal rank. We obtain the second part of Lemma 1 by defining

$$
\left(M^{\prime}(\mathbf{a}) \quad N^{\prime}(\mathbf{a})\right):=\left(\begin{array}{c}
P(\mathbf{a}) M(\mathbf{a}) \\
N(\mathbf{a})
\end{array}\right)^{-1},
$$

where $M^{\prime}$ and $N^{\prime}$ have the dimensions (number of columns) indicated in the statement.

We define

$$
M^{\operatorname{adj}}(\mathbf{a}):=\left(\mathcal{A}_{d}(\mathbf{a}) M^{\prime}(\mathbf{a})\right)^{T}, \quad N^{\operatorname{adj}}(\mathbf{a}):=\left(\mathcal{A}_{d}(\mathbf{a}) N^{\prime}(\mathbf{a})\right)^{T},
$$

so that the following equality holds:

$$
\mathcal{A}_{d}(\mathbf{a})=M^{\operatorname{adj}}(\mathbf{a})^{T} P(\mathbf{a}) M(\mathbf{a})+N^{\operatorname{adj}}(\mathbf{a})^{T} N(\mathbf{a}) .
$$

This is precisely the decomposition obtained in [16]. However, one cannot reproduce Métivier's argument here because the dimensions of matrices do not agree (this is because our shock is undercompressive and so we have additional jump conditions). We have the following result:

LEMMA 2 For all $\mathbf{a} \in \mathcal{U}$, the following decomposition holds:

$$
\mathbb{R}^{2 n}=\operatorname{Ker} M^{\operatorname{adj}}(\mathbf{a}) \oplus \operatorname{Ker} N^{\operatorname{adj}}(\mathbf{a}) .
$$

Proof. Using the definition of the matrices $M^{\prime}$ and $N^{\prime}$, we easily see that the matrix

$$
\left(\begin{array}{l}
M^{\prime}(\mathbf{a})^{T} \\
N^{\prime}(\mathbf{a})^{T}
\end{array}\right)
$$

is invertible. Because the matrix $\mathcal{A}_{d}(\mathbf{a})$ is also invertible, the matrix

$$
\left(\begin{array}{l}
M^{\operatorname{adj}}(\mathbf{a}) \\
N^{\operatorname{adj}}(\mathbf{a})
\end{array}\right)
$$

is invertible (see (19)). The result immediately follows. 
Integrating by parts, one shows that

$$
\forall u, v \in \mathcal{C}_{c}^{\infty}(\Omega), \quad\left\langle\left\langle\mathcal{L}_{\mathbf{a}} u, v\right\rangle\right\rangle_{L^{2}(\Omega)}=\left\langle\left\langle u, \mathcal{L}_{\mathbf{a}}^{*} v\right\rangle\right\rangle_{L^{2}(\Omega)}, \quad\left\langle\left\langle\mathcal{L}_{\mathbf{a}}^{\gamma} u, v\right\rangle_{L^{2}(\Omega)}=\left\langle\left\langle u,\left(\mathcal{L}_{\mathbf{a}}^{\gamma}\right)^{*} v\right\rangle_{L^{2}(\Omega)},\right.\right.
$$

where $\mathcal{L}_{\mathbf{a}}^{*}$ and $\left(\mathcal{L}_{\mathbf{a}}^{\gamma}\right)^{*}$ are given by

$$
\mathcal{L}_{\mathbf{a}}^{*}:=-\sum_{j=0}^{d} \mathcal{A}_{j}(\mathbf{a})^{T} \partial_{j}-\left(\sum_{j=0}^{d} \partial_{j} \mathcal{A}_{j}(\mathbf{a})^{T}\right), \quad\left(\mathcal{L}_{\mathbf{a}}^{\gamma}\right)^{*}:=\mathcal{L}_{\mathbf{a}}^{*}+\gamma \mathcal{A}_{0}(\mathbf{a})^{T} .
$$

We define the dual boundary conditions of $[18]$ in the following way:

$$
N^{\mathrm{adj}}(\mathbf{a}) v=0, \quad \operatorname{div}\left(M_{1}^{\mathrm{adj}}(\mathbf{a}) v\right)=0, \quad x_{d}=0,
$$

where $M_{1}^{\text {adj }}(\mathbf{a})$ is the matrix formed by the first $d$ rows of $M^{\text {adj }}(\mathbf{a})$. In (20), "div" stands for the divergence operator in $\omega=\mathbb{R}^{d}$. The main result of this subsection can be stated as follows:

Proposition 1 Let $\gamma \geqslant 1, \tilde{f} \in L^{2}(\Omega)$ and $\widetilde{g} \in L^{2}(\omega)$. If $(v, \psi) \in L^{2}(\Omega) \times L^{2}(\omega)$ is a weak solution of

$$
\begin{cases}\mathcal{L}_{\mathbf{a}}^{\gamma} v=\tilde{f}, & x_{d}>0 \\ \mathcal{B}_{\mathbf{a}}^{\gamma}(v, \psi)=\tilde{g}, & x_{d}=0\end{cases}
$$

then for all $w \in \mathcal{C}_{c}^{\infty}(\bar{\Omega})$ such that

$$
N^{\operatorname{adj}}(\mathbf{a}) w_{\left.\right|_{x_{d}=0}}=0, \quad \operatorname{div}_{-\gamma}\left(M_{1}^{\mathrm{adj}}(\mathbf{a}) w_{\left.\right|_{x_{d}=0}}\right)=0
$$

we have

$$
\langle\tilde{f}, w\rangle\rangle_{L^{2}(\Omega)}+\left\langle P(\mathbf{a}) \widetilde{g}, M^{\mathrm{adj}}(\mathbf{a}) w\right\rangle_{L^{2}(\omega)}=\left\langle\left\langle v,\left(\mathcal{L}_{\mathbf{a}}^{\gamma}\right)^{*} w\right\rangle\right\rangle_{L^{2}(\Omega)} .
$$

The operator $\operatorname{div}_{-\gamma}$ is defined on $H^{1}\left(\mathbb{R}^{d} ; \mathbb{R}^{d}\right)$ by

$$
\operatorname{div}_{-\gamma} u:=-\gamma u_{0}+\sum_{j=0}^{d-1} \partial_{j} u_{j}
$$

Furthermore, the linear problem

$$
\begin{cases}\mathcal{L}_{\mathbf{a}}^{*} w=0, & x_{d}>0 \\ N^{\operatorname{adj}}(\mathbf{a}) w=0, \quad \operatorname{div}\left(M_{1}^{\operatorname{adj}}(\mathbf{a}) w\right)=0, & x_{d}=0\end{cases}
$$

satisfies the backward uniform Kreiss-Lopatinskii condition, that is, the analogue of the uniform stability condition (Assumption 3) with $\gamma$ changed to $-\gamma$.

Proof. Because $v \in L^{2}(\Omega)$ and $\mathcal{L}_{\mathbf{a}}^{\gamma} v \in L^{2}(\Omega)$, a classical result by Friedrichs ensures that the trace of $v$ on $\omega$ is well defined and belongs to $H^{-1 / 2}(\omega)$. (Here, we use the symmetrizability of the hyperbolic equations, given by Assumption 11) Moreover, the following equality holds for all $w \in \mathcal{C}_{c}^{\infty}(\bar{\Omega})$ :

$$
\left\langle\langle f, w\rangle_{L^{2}(\Omega)}=\left\langle\left\langle v,\left(\mathcal{L}_{\mathbf{a}}^{\gamma}\right)^{*} w\right\rangle_{L^{2}(\Omega)}-\left\langle\mathcal{A}_{d}(\mathbf{a}) v, w\right\rangle_{H^{-1 / 2}(\omega), H^{1 / 2}(\omega)} .\right.\right.
$$


Using the equality

$$
\left(\begin{array}{c}
\nabla^{\gamma} \psi \\
\mathbf{0}
\end{array}\right)+P(\mathbf{a}) M(\mathbf{a}) v=P(\mathbf{a}) \tilde{g} \quad \text { with } \quad \nabla^{\gamma} \psi:=\left(\begin{array}{c}
\gamma \psi+\partial_{0} \psi \\
\partial_{1} \psi \\
\vdots \\
\partial_{d-1} \psi
\end{array}\right),
$$

we obtain

$$
\begin{aligned}
\left\langle\mathcal{A}_{d}(\mathbf{a}) v, w\right\rangle_{H^{-1 / 2}(\omega), H^{1 / 2}(\omega)}= & \left\langle P(\mathbf{a}) \widetilde{g}, M^{\mathrm{adj}}(\mathbf{a}) w\right\rangle_{L^{2}(\omega)}+\left\langle\psi, \operatorname{div}_{-\gamma} M_{1}^{\mathrm{adj}}(\mathbf{a}) w\right\rangle_{L^{2}(\omega)} \\
& +\left\langle N(\mathbf{a}) v, N^{\mathrm{adj}}(\mathbf{a}) w\right\rangle_{H^{-1 / 2}(\omega), H^{1 / 2}(\omega)} .
\end{aligned}
$$

The first part of the proposition follows. We now turn to the study of the boundary value problem

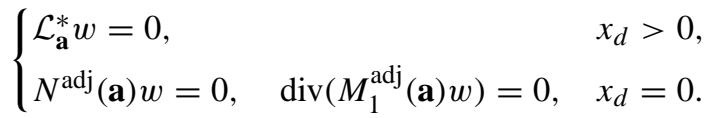

We freeze the coefficients and check the backward uniform Kreiss-Lopatinskii condition. Once the (backward) Laplace transform in $x_{0}$ and the Fourier transform in $x_{1}, \ldots, x_{d-1}$ are performed, the interior equations read

$$
\mathcal{A}_{d}^{T} \frac{\mathrm{d} \widehat{w}}{\mathrm{~d} x_{d}}-\left(\gamma \mathcal{A}_{0}^{T}-i \sum_{j=0}^{d-1} \eta_{j} \mathcal{A}_{j}^{T}\right) \widehat{w}=0, \quad x_{d}>0,
$$

and the boundary conditions read

$$
N^{\mathrm{adj}} \widehat{w}(0)=0, \quad\left(-\gamma+i \eta_{0}, i \eta_{1}, \ldots, i \eta_{d-1}\right) \cdot M_{1}^{\text {adj }} \widehat{w}(0)=0 .
$$

In what follows, we consider the usual hermitian structure on the spaces $\mathbb{C}^{m}$, defined by the hermitian form

$$
\langle X, Y\rangle_{\mathbb{C}^{m}}:=\sum_{i=1}^{m} X_{i} \overline{Y_{i}},
$$

and the sign $\perp$ denotes the orthogonal complement with respect to this hermitian structure. It is proved in [5] that the stable subspace of the matrix

$$
\left(\mathcal{A}_{d}^{T}\right)^{-1}\left(\gamma \mathcal{A}_{0}^{T}-i \sum_{j=0}^{d-1} \eta_{j} \mathcal{A}_{j}^{T}\right)
$$

is equal to $\left(\mathcal{A}_{d} \mathcal{E}^{-}(\eta, \gamma)\right)^{\perp}$. Define $e:=\left(\gamma+i \eta_{0}, i \eta_{1}, \ldots, i \eta_{d-1}\right)^{T}$. We thus need to check that for all $(\eta, \gamma) \in \Sigma$, the subspace

$$
\left\{Z \in\left(\mathcal{A}_{d} \mathcal{E}^{-}(\eta, \gamma)\right)^{\perp}: N^{\mathrm{adj}} Z=0 \text { and }\left\langle M_{1}^{\mathrm{adj}} Z, e\right\rangle_{\mathbb{C}^{d}}=0\right\}
$$

is trivial. Let $Z \in\left(\mathcal{A}_{d} \mathcal{E}^{-}(\eta, \gamma)\right)^{\perp}$ satisfy $Z \in \operatorname{Ker} N^{\text {adj }}$ and

$$
\left\langle M_{1}^{\mathrm{adj}} Z, e\right\rangle_{\mathbb{C}^{d}}=0 .
$$


Let $Y \in \mathbb{C}^{N}$. Because of Assumption 3 , there exist $\chi \in \mathbb{C}$ and $X \in \mathcal{E}^{-}(\eta, \gamma)$ such that

$$
Y=\chi\left(\begin{array}{l}
e \\
\mathbf{0}
\end{array}\right)+P M X
$$

We now compute

$$
\begin{aligned}
\left\langle M^{\text {adj }} Z, Y\right\rangle_{\mathbb{C}^{N}} & =\bar{\chi}\left\langle M_{1}^{\text {adj }} Z, e\right\rangle_{\mathbb{C}^{d}}+\left\langle M^{\text {adj }} Z, P M\right\rangle_{\mathbb{C}^{N}}=\left\langle Z,\left(M^{\text {adj }}\right)^{T} P M X\right\rangle_{\mathbb{C}^{2 n}} \\
& =\left\langle Z, \mathcal{A}_{d} X\right\rangle_{\mathbb{C}^{2 n}}-\left\langle Z,\left(N^{\mathrm{adj}}\right)^{T} N X\right\rangle_{\mathbb{C}^{2 n}}=0 .
\end{aligned}
$$

Because this equality holds for all $Y \in \mathbb{C}^{N}$, we have $Z \in \operatorname{Ker} M^{\text {adj }}$; but we also have $Z \in \operatorname{Ker} N^{\text {adj }}$, and thus $Z=0$ (see Lemma2). This completes the proof.

If $w \in \mathcal{C}_{c}^{\infty}(\bar{\Omega})$ satisfies

$$
N^{\operatorname{adj}}(\mathbf{a}) w_{\left.\right|_{d}=0}=0, \quad \operatorname{div}_{-\gamma}\left(M_{1}^{\operatorname{adj}}(\mathbf{a}) w_{\left.\right|_{x_{d}=0}}\right)=0,
$$

we have the estimate

$$
\gamma\|w\|_{0}^{2}+\left\|w_{\left.\right|_{x_{d}=0}}\right\|_{0}^{2} \leqslant \frac{C(K)}{\gamma}\left\|\left(\mathcal{L}_{\mathbf{a}}^{\gamma}\right)^{*} w\right\|_{0}^{2} .
$$

This estimate is proved as the main estimate of Theorem 2, using Kreiss symmetrizers and paradifferential calculus. We are now able to solve the linearized equations in weighted spaces.

\subsection{Existence of solutions in weighted spaces}

For $\gamma \geqslant 1$, the space $\exp (\gamma t) L^{2}(\Omega)$ is the set of functions $f$ defined on $\Omega$ such that $\exp (-\gamma t) f \in$ $L^{2}(\Omega)$. The space $\exp (\gamma t) H^{s}(\omega)$ is defined in a similar way when $s \in \mathbb{R}$. This subsection is devoted to the proof of the following result:

Proposition 2 Under Assumptions 1,4 there exists $\gamma_{0} \geqslant 1$ such that for all $\gamma \geqslant \gamma_{0}$, for all $f \in \exp (\gamma t) L^{2}(\Omega)$ and for all $g \in \exp (\gamma t) L^{2}(\omega)$, there exists a weak solution $(v, \psi) \in$ $\exp (\gamma t) L^{2}(\Omega) \times \exp (\gamma t) H^{1 / 2}(\omega)$ of the linearized equations

$$
\begin{cases}\mathcal{L}_{\mathbf{a}} v=f, & x_{d}>0, \\ \mathcal{B}_{\mathbf{a}}(v, \psi)=g, & x_{d}=0 .\end{cases}
$$

Proof. Let $F$ denote the subspace

$$
F:=\left\{w \in \mathcal{C}_{c}^{\infty}(\bar{\Omega}): N^{\operatorname{adj}}(\mathbf{a}) w_{\left.\right|_{x_{d}=0}}=0 \text { and } \operatorname{div}_{-\gamma}\left(M_{1}^{\operatorname{adj}}(\mathbf{a}) w_{\left.\right|_{x_{d}}=0}\right)=0\right\}
$$

We let $\tilde{f}:=\exp (-\gamma t) f, \widetilde{g}:=\exp (-\gamma t) g$. We define a linear form on $\left(\mathcal{L}_{\mathbf{a}}^{\gamma}\right)^{*} F$ by the following formula:

$$
\ell\left[\left(\mathcal{L}_{\mathbf{a}}^{\gamma}\right)^{*} w\right]:=\langle\langle\tilde{f}, w\rangle\rangle_{L^{2}(\Omega)}+\left\langle P(\mathbf{a}) \tilde{g}, M^{\operatorname{adj}}(\mathbf{a}) w\right\rangle_{L^{2}(\omega)} .
$$

The linear form $\ell$ is well defined because of $(21)$ and there exists a constant $C>0$ such that

$$
\left|\ell\left[\left(\mathcal{L}_{\mathbf{a}}^{\gamma}\right)^{*} w\right]\right| \leqslant C\left\|\left(\mathcal{L}_{\mathbf{a}}^{\gamma}\right)^{*} w\right\|_{0}
$$


Applying the Hahn-Banach Theorem and then the Riesz Theorem, we can conclude that there exists a function $\widetilde{v} \in L^{2}(\Omega)$ such that for all $w \in F$,

$$
\ell\left[\left(\mathcal{L}_{\mathbf{a}}^{\gamma}\right)^{*} w\right]=\left\langle\left\langle\widetilde{v},\left(\mathcal{L}_{\mathbf{a}}^{\gamma}\right)^{*} w\right\rangle{L^{2}(\Omega)} .\right.
$$

If we first choose test functions $w \in \mathcal{C}_{c}^{\infty}(\Omega)$, we deduce that $\widetilde{v}$ is a weak solution of

$$
\mathcal{L}_{\mathbf{a}}^{\gamma} \widetilde{v}=\tilde{f},
$$

or equivalently, the function $v:=\exp (\gamma t) \widetilde{v}$ is a weak solution in $\exp (\gamma t) L^{2}(\Omega)$ of

$$
\mathcal{L}_{\mathbf{a}} v=f
$$

Using once again Friedrichs's result, we infer that the trace of $\widetilde{v}$ on $\omega$ is well defined and belongs to $H^{-1 / 2}(\omega)$. Furthermore, the following equality holds for all $w \in F$ :

$$
\left\langle\widetilde{v},\left(\mathcal{L}_{\mathbf{a}}^{\gamma}\right)^{*} w\right\rangle_{L^{2}(\Omega)}=\langle\langle\tilde{f}, w\rangle\rangle_{L^{2}(\Omega)}+\left\langle P(\mathbf{a}) M(\mathbf{a}) \widetilde{v}, M^{\mathrm{adj}}(\mathbf{a}) w\right\rangle_{H^{-1 / 2}(\omega), H^{1 / 2}(\omega)},
$$

and combining this equality with (22) yields

$$
\forall w \in F, \quad\left\langle P(\mathbf{a}) M(\mathbf{a}) \widetilde{v}, M^{\operatorname{adj}}(\mathbf{a}) w\right\rangle_{H^{-1 / 2}(\omega), H^{1 / 2}(\omega)}=\left\langle P(\mathbf{a}) \widetilde{g}, M^{\operatorname{adj}}(\mathbf{a}) w\right\rangle_{L^{2}(\omega)} .
$$

By a continuity argument, this equality still holds for all $w \in H^{1}(\Omega)$ such that $N^{\text {adj }}(\mathbf{a}) w=0$ and $\operatorname{div}_{-\gamma}\left(M_{1}^{\mathrm{adj}}(\mathbf{a}) w\right)=0$ on the boundary $\omega$. Note that these equalities make sense because the trace of functions in $H^{1}(\Omega)$ is well defined and belongs to $H^{1 / 2}(\omega)$.

Let $\theta$ be a function in $H^{1 / 2}(\omega)$ that takes its values in $\mathbb{R}^{N}$. We write $\theta=\left(\theta_{1}, \theta_{2}\right)$ where $\theta_{1}$ (resp. $\theta_{2}$ ) takes its values in $\mathbb{R}^{d}$ (resp. $\mathbb{R}^{N-d}$ ). Then there exists a function $w \in H^{1}(\Omega)$ such that

$$
N^{\mathrm{adj}}(\mathbf{a}) w_{\left.\right|_{x_{d}}=0}=0, \quad M^{\mathrm{adj}}(\mathbf{a}) w_{\left.\right|_{x_{d}}=0}=\theta .
$$

This classical lifting argument works because both matrices $N^{\text {adj }}(\mathbf{a})$ and $M^{\text {adj }}(\mathbf{a})$ are Lipschitzean. Consequently, for all $\theta_{2} \in H^{1 / 2}\left(\omega ; \mathbb{R}^{N-d}\right)$, we have

$$
\left\langle P(\mathbf{a}) M(\mathbf{a}) \widetilde{v},\left(0, \theta_{2}\right)\right\rangle_{H^{-1 / 2}(\omega), H^{1 / 2}(\omega)}=\left\langle P(\mathbf{a}) \widetilde{g},\left(0, \theta_{2}\right)\right\rangle_{L^{2}(\omega)},
$$

and for all $\theta_{1} \in H^{1 / 2}\left(\omega ; \mathbb{R}^{d}\right)$ satisfying $\operatorname{div}_{-\gamma} \theta_{1}=0$, we have

$$
\left\langle P(\mathbf{a}) M(\mathbf{a}) \widetilde{v},\left(\theta_{1}, 0\right)\right\rangle_{H^{-1 / 2}(\omega), H^{1 / 2}(\omega)}=\left\langle P(\mathbf{a}) \widetilde{g},\left(\theta_{1}, 0\right)\right\rangle_{L^{2}(\omega)} .
$$

The result of the proposition follows from the following lemma:

LEMma 3 Let $\gamma \geqslant 1$ and let $u \in H^{-1 / 2}\left(\mathbb{R}^{d} ; \mathbb{R}^{d}\right)$ be such that for all $w \in \mathcal{C}_{c}^{\infty}\left(\mathbb{R}^{d} ; \mathbb{R}^{d}\right)$, one has

$$
\operatorname{div}_{-\gamma} w=0 \Rightarrow\langle u, w\rangle_{H^{-1 / 2}, H^{1 / 2}}=0 .
$$

Then there exists $\psi \in H^{1 / 2}\left(\mathbb{R}^{d} ; \mathbb{R}\right)$ such that

$$
u=\nabla^{\gamma} \psi=\left(\begin{array}{c}
\gamma \psi+\partial_{0} \psi \\
\partial_{1} \psi \\
\vdots \\
\partial_{d-1} \psi
\end{array}\right)
$$

Furthermore, the solution $\psi$ satisfies $\|\psi\|_{1 / 2, \gamma} \leqslant\|u\|_{-1 / 2, \gamma}$. 
Applying Lemma 3, we can conclude that there exists $\widetilde{\psi} \in H^{1 / 2}\left(\mathbb{R}^{d}\right)$ such that

$$
\left(\begin{array}{c}
\nabla^{\gamma} \widetilde{\psi} \\
\mathbf{0}
\end{array}\right)+P(\mathbf{a}) M(\mathbf{a}) \widetilde{v}=P(\mathbf{a}) \widetilde{g} .
$$

Proposition 2 follows by setting $\psi:=\exp (\gamma t) \widetilde{\psi}$.

We refer to [7, 17] for the proof of Lemma 3, which is a very classical result.

\subsection{Final steps in the analysis}

Proposition 2 asserts the existence of weak solutions as long as the data belong to weighted spaces, but we have said nothing yet about the uniqueness of weak solutions and continuity with respect to the data. The lack of regularity of weak solutions makes it impossible to use our main energy estimate (Theorem 22). The next step consists in showing that weak solutions exhibited in Proposition 2 are strong solutions, in the following sense: there exist sequences $\left(v^{\nu}\right)$ in $\exp (\gamma t) H^{1}(\Omega)$ and $\left(\psi^{\nu}\right)$ in $\exp (\gamma t) H^{1}(\omega)$ such that

$$
\begin{aligned}
\exp (-\gamma t) v^{v} \rightarrow \exp (-\gamma t) v & \text { in } L^{2}(\Omega), \\
\mathcal{L}_{\mathbf{a}}^{\gamma}\left(\exp (-\gamma t) v^{\nu}\right) \rightarrow \widetilde{f} & \text { in } L^{2}(\Omega), \\
\exp (-\gamma t) v_{\left.\right|_{d}=0}^{v} \rightarrow \exp (-\gamma t) v_{\left.\right|_{d}=0} & \text { in } H^{-1 / 2}\left(\mathbb{R}^{d}\right), \\
\exp (-\gamma t) \psi^{v} \rightarrow \exp (-\gamma t) \psi & \text { in } H^{1 / 2}\left(\mathbb{R}^{d}\right), \\
\mathcal{B}_{\mathbf{a}}^{\gamma}\left(\exp (-\gamma t) v^{v}, \exp (-\gamma t) \psi^{\nu}\right) \rightarrow \widetilde{g} & \text { in } L^{2}\left(\mathbb{R}^{d}\right) .
\end{aligned}
$$

Furthermore $\left(\exp (-\gamma t) v^{v}, \exp (-\gamma t) \psi^{\nu}\right)$ satisfies the energy estimate (17). Using the convergences stated above, we conclude that $\exp (-\gamma t) \psi \in H^{1}\left(\mathbb{R}^{d}\right)$ and $\exp (-\gamma t) v_{\left.\right|_{d}=0} \in L^{2}\left(\mathbb{R}^{d}\right)$, and $(\exp (-\gamma t) v, \exp (-\gamma t) \psi)$ satisfies the energy estimate (17). In particular, we have a uniqueness result in weighted spaces. We refer to [16, 17] for the details (the regularized sequence is obtained via tangential mollifiers).

Continuing the analysis of the linearized equations, one shows that for data vanishing in the past $(f=0$ and $g=0$ for $t<0$ ), solutions also vanish in the past and one can then solve the boundary value problem (18) supplemented with an initial condition. The arguments are similar to those in [5, 16]. This extends the previous work by Rauch [18] that dealt with $\mathcal{C}^{\infty}$ coefficients. We can summarize the main result on the linear problem in the following way:

Theorem 3 Under Assumptions 14 , consider the data

$$
f \in L^{2}(] 0, T\left[\times \mathbb{R}_{+}^{d}\right), \quad g \in L^{2}(] 0, T\left[\times \mathbb{R}^{d-1}\right), \quad v_{0} \in L^{2}\left(\mathbb{R}_{+}^{d}\right), \quad \psi_{0} \in H^{1 / 2}\left(\mathbb{R}^{d-1}\right),
$$

where $T$ is a fixed positive number. Then the linearized problem

$$
\begin{aligned}
\sum_{j=0}^{d} \mathcal{A}_{j}(\mathbf{a}) \partial_{j} v & \left.=f, \quad x_{d}>0, t \in\right] 0, T[, \\
\sum_{j=0}^{d-1} \partial_{j} \psi b_{j}(\mathbf{a})+M(\mathbf{a}) v & \left.=g, \quad x_{d}=0, t \in\right] 0, T[,
\end{aligned}
$$


supplemented with the initial condition

$$
v_{\left.\right|_{t=0}}=v_{0}, \quad \psi_{\left.\right|_{t=0}}=\psi_{0},
$$

has a unique solution $(v, \psi) \in L^{2}(] 0, T\left[\times \mathbb{R}_{+}^{d}\right) \times H^{1}(] 0, T\left[\times \mathbb{R}^{d-1}\right)$ such that $v \in$ $\mathcal{C}\left([0, T] ; L^{2}\left(\mathbb{R}_{+}^{d}\right)\right)$ and for all $\gamma \geqslant \gamma_{0}$ and all $t \in[0, T]$, the following estimate holds:

$$
\begin{aligned}
& e^{-2 \gamma t}\|v(t)\|_{L^{2}\left(\mathbb{R}_{+}^{d}\right)}^{2}+\gamma\left\|e^{-\gamma s} v\right\|_{L^{2}\left(\left[0, t\left[\times \mathbb{R}_{+}^{d}\right)\right.\right.}^{2}+\left\|e^{-\gamma s} v_{\left.\right|_{x_{d}=0}}\right\|_{L^{2}(] 0, t\left[\times \mathbb{R}^{d-1}\right)}^{2}+\left\|e^{-\gamma s} \psi\right\|_{H^{1}(] 0, t\left[\times \mathbb{R}^{d-1}\right)}^{2} \\
& \leqslant C\left(\frac{1}{\gamma}\left\|e^{-\gamma s} f\right\|_{L^{2}(] 0, t\left[\times \mathbb{R}_{+}^{d}\right)}^{2}+\left\|e^{-\gamma s} g\right\|_{L^{2}(] 0, t\left[\times \mathbb{R}^{d-1}\right)}^{2}+\left\|v_{0}\right\|_{L^{2}\left(\mathbb{R}_{+}^{d}\right)}^{2}+\left\|\psi_{0}\right\|_{H^{1 / 2}\left(\mathbb{R}^{d-1}\right)}^{2}\right) .
\end{aligned}
$$

The constants $C$ and $\gamma_{0}$ are independent of $t, T, f, g$, but depend on the constant $K>0$ and on the compact set $\mathcal{K}$ (see Theorem 2).

In order to construct an iteration scheme to solve the nonlinear equations, we need to know more about the regularity of the solutions to the linear problem. The main question is: given data that belong to higher order Sobolev spaces, does the solution also belong to a higher order Sobolev space? The question was addressed in [19] and the answer is yes provided that a number of compatibility conditions hold. These compatibility conditions are satisfied when the initial data $v_{0}$ and $\psi_{0}$ are zero, and the source terms $f$ and $g$ vanish in the past.

To obtain an energy estimate in a Sobolev space, one first commutes the linearized equations with tangential derivatives and uses Theorem 3 (as well as Gagliardo-Nirenberg inequalities to estimate commutators). The normal derivatives are directly estimated by using the equation

$$
\partial_{d} v=\mathcal{A}_{d}(\mathbf{a})^{-1} f-\sum_{j=0}^{d-1} \mathcal{A}_{d}(\mathbf{a})^{-1} \mathcal{A}_{j}(\mathbf{a}) \partial_{j} v .
$$

This is possible because the interface is noncharacteristic (Assumption 2), so one can reproduce Métivier's analysis (see [16]). Finally, let us assume that $\dot{u} \in H^{s}\left(\Omega_{T}\right)$ and $\varphi \in H^{s+1}\left(\omega_{T}\right)$, where $\left.\left.\left.\left.\Omega_{T}:=\right]-\infty, T\right] \times \mathbb{R}_{+}^{d}, \omega_{T}:=\right]-\infty, T\right] \times \mathbb{R}^{d-1}, s>(d+3) / 2$, and $(\dot{u}, \varphi)$ vanish for $t<T_{0}$. (Recall that $(\dot{u}, \varphi)$ is the perturbation of the planar interface about which the equations are linearized). Then, if $f \in H^{s}\left(\Omega_{T}\right)$ and $g \in H^{s}\left(\omega_{T}\right)$ vanish in the past, the solution $(v, \psi)$ to the linearized system (18) satisfies $v \in H^{s}\left(\Omega_{T}\right), \psi \in H^{s+1}\left(\omega_{T}\right)$, and the energy estimate

$$
\begin{aligned}
\gamma\|v\|_{H_{\gamma}^{s}\left(\Omega_{T}\right)}^{2}+ & \left\|v_{\left.\right|_{x_{d}=0}}\right\|_{H_{\gamma}^{s}\left(\omega_{T}\right)}^{2}+\|\psi\|_{H_{\gamma}^{s+1}\left(\omega_{T}\right)}^{2} \\
\leqslant & C(K)\left(\frac{1}{\gamma}\|f\|_{H_{\gamma}^{s}\left(\Omega_{T}\right)}^{2}+\|g\|_{H_{\gamma}^{s}\left(\omega_{T}\right)}^{2}\right) \\
& \quad \frac{C(K)}{\gamma}\left(1+\|v\|_{W^{1, \infty}\left(\Omega_{T}\right)}^{2}+\|f\|_{L^{\infty}\left(\Omega_{T}\right)}^{2}\right)\left(\|\dot{u}\|_{H_{\gamma}^{s}\left(\Omega_{T}\right)}^{2}+\|\varphi\|_{H_{\gamma}^{s+1}\left(\omega_{T}\right)}^{2}\right) .
\end{aligned}
$$

Here, we have kept the notations of [16]: for instance, $H_{\gamma}^{s}\left(\Omega_{T}\right)$ denotes the space $\exp (\gamma t) H^{s}\left(\Omega_{T}\right)$ and is equipped with the norm

$$
\|v\|_{H_{\gamma}^{s}\left(\Omega_{T}\right)}:=\sum_{|\alpha| \leqslant s} \gamma^{s-|\alpha|}\left\|\exp (-\gamma t) \partial^{\alpha} v\right\|_{L^{2}\left(\Omega_{T}\right)} .
$$


We now describe the iteration scheme used to solve the nonlinear equations (7). In [13], Majda used a classical Picard iteration scheme

$$
\begin{aligned}
& L\left(u_{+}^{v}, \varphi^{v}\right)\left(u_{+}^{v+1}-u_{+}^{\mathrm{app}}\right)=-L\left(u_{+}^{v}, \varphi^{v}\right) u_{+}^{\mathrm{app}}, \\
& L\left(u_{-}^{v}, \varphi^{v}\right)\left(u_{-}^{v+1}-u_{-}^{\mathrm{app}}\right)=-L\left(u_{-}^{\nu}, \varphi^{\nu}\right) u_{-}^{\mathrm{app}},
\end{aligned}
$$

for the interior equations, together with a Newton scheme for the boundary conditions. In (23), the functions $\left(u_{+}^{\text {app }}, u_{-}^{\text {app }}\right)$ are an approximate solution constructed with compatible initial conditions (see [14, 16]). In [16], Métivier first reduces the nonlinear boundary conditions to linear boundary conditions. This is made possible by the uniform stability condition but it heavily uses the particular form of the Rankine-Hugoniot conditions. It can therefore hardly apply in our framework because of the additional jump conditions. We thus go back to Majda's method which was also used in [17]. The scheme used for the interior equations is defined by 23$)$ and the solution $\left(u_{+}^{v+1}-u_{+}^{\text {app }}\right.$, $u_{-}^{v+1}-u_{-}^{\text {app }}$ ) is sought as a function vanishing for $t<0$. (The approximate solution $u^{\text {app }}$ equals the initial condition at $t=0$ ). This is made possible by the arguments developed when solving the linearized problems. As said previously, the nonlinear boundary conditions are solved by a Newton method. Recall that we look for $u_{+}, u_{-}$and $\varphi$ that satisfy $B\left(u_{+}, u_{-}, \varphi\right)=0$ on $\left\{x_{d}=0\right\}$, so the Newton method reads

$$
d B\left(u_{+}^{v}, u_{-}^{v}, \varphi^{v}\right) \cdot\left(u_{+}^{v+1}-u_{+}^{v}, u_{-}^{v+1}-u_{-}^{v}, \varphi^{v+1}-\varphi^{v}\right)=-B\left(u_{+}^{v}, u_{-}^{v}, \varphi^{v}\right) .
$$

Using $(8 \mathrm{c})$ and the definition of the linearized operator $\mathcal{B}$ (see $(14 \mathrm{~b})$ ), we may rewrite $(24)$ as

$$
\begin{aligned}
\mathcal{B}_{\mathbf{a}^{v}}\left(u_{+}^{v+1}-u_{+}^{\mathrm{app}}, u_{-}^{v+1}-u_{-}^{\mathrm{app}}, \varphi^{\nu+1}-\varphi^{\mathrm{app}}\right) & \\
& =\mathcal{B}_{\mathbf{a}^{v}}\left(u_{+}^{\nu}-u_{+}^{\mathrm{app}}, u_{-}^{\nu}-u_{-}^{\mathrm{app}}, \varphi^{\nu}-\varphi^{\mathrm{app}}\right)-B\left(u_{+}^{v}, u_{-}^{v}, \varphi^{\nu}\right),
\end{aligned}
$$

with $\mathbf{a}^{v}:=\left(u_{+}^{v}, u_{-}^{v}, \varphi^{v}\right)$. At each step of the iteration scheme, the initial conditions are

$$
\left(u_{+}^{\nu+1}-u_{+}^{\mathrm{app}}, u_{-}^{\nu+1}-u_{-}^{\mathrm{app}}, \varphi^{\nu+1}-\varphi^{\mathrm{app}}\right)_{\left.\right|_{t=0}}=0 .
$$

Using this iteration scheme, we end up with the main result of this paper:

THEOREM 4 Fix an integer $s>(d+3) / 2$, and initial data $\left(u_{+}^{0}, u_{-}^{0}, \varphi^{0}\right)$ satisfying

$$
u_{+}^{0}-u_{r} \in H^{s+1 / 2}\left(\mathbb{R}_{+}^{d}\right), \quad u_{-}^{0}-u_{l} \in H^{s+1 / 2}\left(\mathbb{R}_{+}^{d}\right), \quad \varphi^{0} \in H^{s+1 / 2}\left(\mathbb{R}^{d-1}\right),
$$

such that $u_{+}^{0}-u_{r}, u_{-}^{0}-u_{l}$ and $\varphi^{0}$ have compact support and satisfy the natural compatibility conditions up to order $s-1$. Assume also that $\left(u_{+}^{0}-u_{r}, u_{-}^{0}-u_{l}, \varphi^{0}\right)$ takes its values in a compact subset of $\mathcal{U}$. Then there exists a unique smooth solution $\left(u_{+}, u_{-}, \varphi\right)$ of (7) defined on a time interval $[0, T]$ with $T>0$. Furthermore, the solution $\left(u_{+}, u_{-}, \varphi\right)$ satisfies

$$
u_{+}-u_{r} \in H^{s}(] 0, T\left[\times \mathbb{R}_{+}^{d}\right), \quad u_{-}-u_{l} \in H^{s}(] 0, T\left[\times \mathbb{R}_{+}^{d}\right), \quad \varphi \in H^{s+1}(] 0, T\left[\times \mathbb{R}^{d-1}\right) .
$$

Recall that when the initial data satisfy the regularity assumptions of Theorem 4, and are compatible up to order $s-1$, one can construct an approximate solution $\left(u_{+}^{\text {app }}, u_{-}^{\text {app }}, \varphi^{\text {app }}\right)$ that satisfies

$$
u_{+}^{\text {app }}-u_{r} \in H^{s+1}(\Omega), \quad u_{-}^{\text {app }}-u_{l} \in H^{s+1}(\Omega), \quad \varphi^{\text {app }} \in H^{s+1}\left(\mathbb{R}^{d-1}\right),
$$


and

$$
\forall j=0, \ldots, s-1, \quad\left\{\begin{array}{l}
\partial_{t}^{j}\left[L\left(u_{ \pm}^{\text {app }}, \varphi^{\text {app }}\right) u_{ \pm}^{\text {app }}\right]_{t=0}=0, \\
\partial_{t}^{j} B\left(u_{+}^{\text {app }}, u_{-}^{\text {app }}, \varphi^{\text {app }}\right)=0 .
\end{array}\right.
$$

We refer to [16,7] for the proof of Theorem 4 The idea is to prove that for the iteration scheme defined above, the sequence $\left(u^{v}, \nabla \varphi^{\nu}\right)$ is bounded in $H^{s}$ and converges in $L^{2}$. Then one shows that the limit is actually a solution in $H^{s}$.

\section{Isothermal phase transitions}

In this section, we show how our analysis applies to the case of isothermal phase transitions in a van der Waals fluid. We refer to [2] for the proofs of the result stated below. We are interested here in the isothermal Euler equations

$$
\left\{\begin{array}{l}
\partial_{t} \rho+\nabla \cdot(\rho \mathbf{u})=0 \\
\partial_{t}(\rho \mathbf{u})+\nabla \cdot(\rho \mathbf{u} \otimes \mathbf{u})+\nabla p(\rho)=0 .
\end{array}\right.
$$

As usual, $\rho$ stands for the density of the fluid, $\mathbf{u}$ for the velocity, $p$ for the pressure. It is a well known fact that this system meets Assumption 1 in the region where it is hyperbolic, that is, in the region $\left\{p^{\prime}(\rho)>0\right\}$. In the following, $v$ stands for the specific volume of the fluid, $v:=1 / \rho$. We assume that the fluid obeys a van der Waals pressure law

$$
p(\rho)=P(v)=\frac{R T}{v-b}-\frac{a}{v^{2}} .
$$

The parameters $a$ and $b$ are positive and $R$ stands for the perfect gas constant. When $T$ is below the critical temperature $T_{c}:=8 a /(27 b R)$, the isothermal curve $(v \mapsto P(v))$ is nonmonotone and we thus expect phase boundaries to propagate. More precisely, $P$ decreases but on an interval $] v_{*}, v^{*}[$. The interval $] b, v_{*}[$ (resp. $] v^{*}, \infty[$ ) is referred to as the liquid (resp. vapor) phase. Another important feature of the pressure law $P$ is the existence and uniqueness of the so-called Maxwell equilibrium points $\left(v_{m}, v^{m}\right)$. These are defined by

$$
v_{m}<v_{*}<v^{*}<v^{m}, \quad P\left(v_{m}\right)=P\left(v^{m}\right)=: \bar{P}, \quad \int_{v_{m}}^{v^{m}}(\bar{P}-P(v)) \mathrm{d} v=0 .
$$

We let

$$
U:= \begin{cases}\left(\rho_{r}, \mathbf{u}_{r}\right) & \text { if } \mathbf{x} \cdot \mathbf{n}>\sigma t, \\ \left(\rho_{l}, \mathbf{u}_{l}\right) & \text { if } \mathbf{x} \cdot \mathbf{n}<\sigma t,\end{cases}
$$

be a weak solution of the Euler equations with $1 / \rho_{r}$ and $1 / \rho_{l}$ close to Maxwell equilibrium points $\left(v_{m}, v^{m}\right)$ (for instance $1 / \rho_{l}$ close to $v_{m}$ and $1 / \rho_{r}$ close to $v^{m}$ ). Without loss of generality, we may assume that the following inequalities hold:

$$
\mathbf{u}_{r} \cdot \mathbf{n}-c_{r}<\sigma<\mathbf{u}_{r} \cdot \mathbf{n}, \quad \mathbf{u}_{l} \cdot \mathbf{n}-c_{l}<\sigma<\mathbf{u}_{l} \cdot \mathbf{n},
$$

where $c$ is the sound speed in the fluid (recall that $c$ is given by $c(\rho):=\sqrt{p^{\prime}(\rho)}$ ). This corresponds to an undercompressive shock wave with $\kappa=0$ (see (5) and Definition 1]. Following Slemrod [24], we shall say that $U$ is an admissible phase transition if there exists a traveling wave

$$
\rho(t, \mathbf{x})=\widetilde{\rho}((\mathbf{x} \cdot \mathbf{n}-\sigma t) / \varepsilon), \quad \mathbf{u}(t, \mathbf{x})=\widetilde{\mathbf{u}}((\mathbf{x} \cdot \mathbf{n}-\sigma t) / \varepsilon)
$$


satisfying

$$
\widetilde{\rho}(+\infty)=\rho_{r}, \tilde{\rho}(-\infty)=\rho_{l}, \quad \widetilde{\mathbf{u}}(+\infty)=\mathbf{u}_{r}, \widetilde{\mathbf{u}}(-\infty)=\mathbf{u}_{l},
$$

that is a solution to the Euler equations supplemented with a capillarity coefficient $\varepsilon^{2}$ and a viscocity coefficient $\nu \varepsilon(\nu>0)$ :

$$
\begin{aligned}
& \partial_{t} \rho+\nabla \cdot(\rho \mathbf{u})=0, \\
& \partial_{t}(\rho \mathbf{u})+\nabla \cdot(\rho \mathbf{u} \otimes \mathbf{u})+\nabla p(\rho)=v \varepsilon \Delta \mathbf{u}-\varepsilon^{2} \nabla(\Delta v) .
\end{aligned}
$$

Let $F$ denote the specific free energy of the fluid, $F^{\prime}(v)=-P(v)$. It is shown in [2] that admissible phase transitions satisfy the Rankine-Hugoniot conditions together with the additional (nonexplicit) jump condition

$$
[F]+[v] \frac{P\left(v_{r}\right)+P\left(v_{l}\right)}{2}=-v j \int_{\mathbb{R}} \widetilde{v}^{\prime}(\xi)^{2} \mathrm{~d} \xi
$$

where

$$
j:=\rho_{r}\left(\mathbf{u}_{r} \cdot \mathbf{n}-\sigma\right)=\rho_{l}\left(\mathbf{u}_{l} \cdot \mathbf{n}-\sigma\right)
$$

denotes the mass transfer across the discontinuity. On account of the Rankine-Hugoniot conditions, the additional jump condition can be rewritten as

$$
\left[f^{\prime}(\rho)+\frac{1}{2}(\mathbf{u} \cdot \mathbf{n}-\sigma)^{2}\right]=-v j \int_{\mathbb{R}} \widetilde{v}^{\prime}(\xi)^{2} \mathrm{~d} \xi,
$$

where $f(\rho):=\rho F(1 / \rho)$ stands for the free energy per unit volume. We have thus a (nonexplicit) form for the function $h$ introduced in Definition 2. The reader will note that the function $h$ depends on $v>0$. Therefore, there is a very wide choice of admissibility criteria. The following theorem is proved in [2] (we refer to this paper for a precise statement):

THEOREM 5 (Benzoni-Gavage [2]) For sufficiently small viscosity coefficient $v$, there exist admissible planar phase transitions, and such phase transitions are linearly uniformly stable, that is, meet Assumption 3 The space dimension is either $d=2$ or $d=3$.

In order to apply the analysis developed in this paper, we only need to check Assumption 4. since we already know that Assumptions 13 are satisfied. Performing a change of observer, we may assume that $\sigma=0, \mathbf{n}=(0, \ldots, 0,1)$ and that the velocities on either side of the shock are parallel to $\mathbf{n}$, that is, $\mathbf{u}_{r, l}=\left(0, \ldots, 0, u_{r, l}\right)$.

For the particular system of Euler's equations supplemented with the above additional jump condition, it is proved in [2] that the matrix $M$ involved in the linearized boundary conditions is given by

$$
M=\left(\begin{array}{cccccc}
u_{r} & \mathbf{0} & \rho_{r} & -u_{l} & \mathbf{0} & -\rho_{l} \\
\mathbf{0} & \rho_{r} u_{r} I_{d-1} & \mathbf{0} & \mathbf{0} & -\rho_{l} u_{l} I_{d-1} & \mathbf{0} \\
u_{r}^{2}+c_{r}^{2} & \mathbf{0} & 2 \rho_{r} u_{r} & -\left(u_{l}^{2}+c_{l}^{2}\right) & \mathbf{0} & -2 \rho_{l} u_{l} \\
f^{\prime \prime}\left(\rho_{r}\right) & \mathbf{0} & u_{r} & \tilde{v} u_{l}-f^{\prime \prime}\left(\rho_{l}\right) & \mathbf{0} & \tilde{v} \rho_{l}-u_{l}
\end{array}\right),
$$

where $\tilde{v}=\alpha \nu+o(v), \alpha$ being a positive constant. Recall that $f$ is the free energy per unit volume. In order to check that $M$ has maximal rank, we are going to show that the submatrix $M_{1}$ consisting of the first $d+1$ columns and of the last column of $M$ is invertible. We have

$$
f^{\prime \prime}(\rho)=\frac{1}{\rho} p^{\prime}(\rho)=\frac{c^{2}}{\rho},
$$


and therefore

$$
M_{1}=\left(\begin{array}{cccc}
u_{r} & \mathbf{0} & \rho_{r} & -\rho_{l} \\
\mathbf{0} & \rho_{r} u_{r} I_{d-1} & \mathbf{0} & \mathbf{0} \\
u_{r}^{2}+c_{r}^{2} & \mathbf{0} & 2 \rho_{r} u_{r} & -2 \rho_{l} u_{l} \\
c_{r}^{2} / \rho_{r} & \mathbf{0} & u_{r} & \tilde{v} \rho_{l}-u_{l}
\end{array}\right) .
$$

The determinant of $M_{1}$ is given by

$$
\operatorname{det} M_{1}=\left(\rho_{r} u_{r}\right)^{d-1}\left(u_{r}^{2}-c_{r}^{2}\right)\left([u][\rho]+\tilde{v} \rho_{r} \rho_{l}\right),
$$

so $M_{1}$ is an invertible matrix for sufficiently small viscosity coefficient $v$. Assumption 4 is therefore met and we can apply the existence result of Section 5 to these admissible phase transitions.

\section{Acknowledgments}

I thank Sylvie Benzoni-Gavage and Guy Métivier for helpful and stimulating discussions on the subject. Part of this work was achieved during a visit at Rennes University. It is my pleasure to thank the Mathematics Department of Rennes University for its hospitality during this visit.

\section{REFERENCES}

1. Benzoni-Gavage, S. Stability of multi-dimensional phase transitions in a van der Waals fluid. Nonlinear Anal. 31 (1998), 243-263. Zbl 0928.76015

2. Benzoni-Gavage, S. Stability of subsonic planar phase boundaries in a van der Waals fluid. Arch. Rat. Mech. Anal. 150 (1999), 23-55. Zbl 0980.76023

3. Benzoni-Gavage, A. \& Serre, D. First order systems of hyperbolic partial differential equations with applications. In preparation.

4. Blokhin, A. M. Strong Discontinuities in Magnetohydrodynamics. Nova Science Publ. (1994). Zbl 0920.76001

5. Chazarain, J. \& Piriou, A. Introduction to the Theory of Linear Partial Differential Equations. North-Holland, Amsterdam, 1982. Zbl 0487.35002 MR 83j:35001

6. Colombo, R. \& Corli, A. Continuous dependence in conservation laws with phase transitions. SIAM J. Math. Anal. 31 (1999), 34-62. Zbl 0977.35085 MR 2000h:35100

7. Coulombel, J. F. Stabilité multidimensionnelle d'interfaces dynamiques. Application aux transitions de phase liquide-vapeur. PhD thesis. ÉNS Lyon (2002).

8. FreistüHLeR, H. The persistence of ideal shock waves. Appl. Math. Lett. 7 (1994), 7-11. Zbl 0811.35078 MR 96c:35114

9. FreistüHLER, H. A short note on the persistence of ideal shock waves. Arch. Math. (Basel) 64 (1995), 344-352. Zbl 0820.35092 MR 96i:35084

10. FreistüHLER, H. Some results on the stability of non-classical shock waves. J. Partial Differential Equations 11 (1998), 25-38. Zbl 0903.35006 MR 99e:35142

11. Hersh, R. Mixed problems in several variables. J. Math. Mech. 12 (1963), 317-334. Zbl 0149.06602 MR $26 \# 5304$

12. KreIss, H. O. Initial boundary value problems for hyperbolic systems. Comm. Pure Appl. Math. 23 (1970), 277-298. Zbl 0193.0690 MR 55 \#10862

13. Majda, A. The existence of multi-dimensional shock fronts. Mem. Amer. Math. Soc. 281 (1983). Zbl 0517.76068 MR 85f:35139 
14. MajdA, A. The stability of multi-dimensional shock fronts. Mem. Amer. Math. Soc. 275 (1983). Zbl 0506.76075 MR 84e:35100

15. MajdA, A. Compressible Fluid Flow and Systems of Conservation Laws in Several Space Variables. Springer, New York (1984). Zbl 0537.76001 MR 85e:35077

16. MÉTIVIER, G. Stability of multidimensional shocks. Advances in the Theory of Shock Waves, Birkhäuser (2001), 25-103. Zbl pre01664415 MR 2002m:35148

17. Mokrane, A. Problèmes mixtes hyperboliques non-linéaires. Ph.D. thesis, Université de Rennes I (1987).

18. RAUCH, J. $L^{2}$ is a continuable initial condition for Kreiss' mixed problems. Comm. Pure Appl. Math. 25 (1972), 265-285. Zbl 0226.35056 MR 45 \#7284

19. RAUCh, J. \& MASSEY III, F. J. Differentiability of solutions to hyperbolic initial-boundary value problems. Trans. Amer. Math. Soc. 189 (1974), 303-318. Zbl 0282.35014 MR 49\#5582

20. SERre, D. Systems of Conservation Laws. 1. Cambridge Univ. Press, Cambridge (1999). Zbl 0930.35001 MR 2000g:35142

21. Serre, D. Systems of Conservation Laws. 2. Cambridge Univ. Press, Cambridge (2000). Zbl 0936.35001 MR 2001c:35146

22. SheARER, M. Admissibility criteria for shock wave solutions of a system of conservation laws of mixed type. Proc. Roy. Soc. Edinburgh Sect. A 93 (1982/83), 233-244. Zbl 0511.35059 MR 84c:35075

23. Shearer, M. Nonuniqueness of admissible solutions of Riemann initial value problems for a system of conservation laws of mixed type. Arch. Rat. Mech. Anal. 93 (1986), 45-59. Zbl 0613.35048 MR 87h:35207

24. Slemrod, M. Admissibility criteria for propagating phase boundaries in a van der Waals fluid. Arch. Rat. Mech. Anal. 81 (1983), 301-315. Zbl 0505.76082 MR 84a:76030

25. TRUSKINOVSKY, L. About the "normal growth" approximation in the dynamical theory of phase transitions. Contin. Mech. Thermodyn. 6 (1994), 185-208. Zbl 0877.73006 MR 95c:80006 